\title{
Organic Matter in the Solar System-Implications for Future on-Site and Sample Return Missions
}

\author{
Zita Martins ${ }^{1}$ D . Queenie Hoi Shan Chan ${ }^{2,3}$. \\ Lydie Bonal $^{4}$ - Ashley King ${ }^{2,5}$ • Hikaru Yabuta ${ }^{6}$
}

Received: 9 July 2019 / Accepted: 23 April 2020 / Published online: 12 May 2020

(C) The Author(s) 2020

\begin{abstract}
Solar system bodies like comets, asteroids, meteorites and dust particles contain organic matter with different abundances, structures and chemical composition. This chapter compares the similarities and differences of the organic composition in these planetary bodies. Furthermore, these links are explored in the context of detecting the most pristine organic material, either by on-site analysis or sample return missions. Finally, we discuss the targets of potential future sample return missions, as well as the contamination controls that should be in place in order to successfully study pristine organic matter.
\end{abstract}

Keywords Organic matter · Solar system · Comets · Asteroids · Extraterrestrial samples · Sample return mission

Role of Sample Return in Addressing Major Questions in Planetary Sciences

Edited by Mahesh Anand, Sara Russell, Yangting Lin, Meenakshi Wadhwa, Kuljeet Kaur Marhas and Shogo Tachibana

\section{Z. Martins}

zita.martins@tecnico.ulisboa.pt

1 Centro de Química Estrutural, Departamento de Engenharia Química, Instituto Superior Técnico, Universidade de Lisboa, Avenida Rovisco Pais 1, 1049-001 Lisboa, Portugal

2 Planetary and Space Sciences, School of Physical Sciences, The Open University, Walton Hall, Milton Keynes MK7 6AA, UK

3 Present address: Department of Earth Sciences, Royal Holloway University of London, Egham, Surrey TW20 0EX, UK

4 Institut de Planétologie et d'Astrophysique de Grenoble, Univ. Grenoble Alpes, CNRS, CNES, 38000 Grenoble, France

5 The Natural History Museum, Cromwell Road, London SW7 5BD, UK

6 Department of Earth and Planetary Systems Science, Hiroshima University, 1-3-1 Kagamiyama, Hiroshima 739-8526, Japan 


\section{Introduction}

Organic matter is widespread throughout the solar system, and the access to its primitive form is achieved by analysing extraterrestrial samples from comets (Bockelée-Morvan et al. 2019), asteroids, meteorites and dust particles. Comets contain a wide variety of extraterrestrial molecules, including glycine and a significant number of carbon-rich species (Altwegg et al. 2016; Bockelée-Morvan et al. 2004; Crovisier and Bockelée-Morvan 1999; Crovisier et al. 2009; Elsila et al. 2009; Goesmann et al. 2015; Mumma and Charnley 2011; Wright et al. 2015). Dust particles, such as micrometeorites, interplanetary dust particles (IDPs), and Ultra-carbonaceous Antarctic Micrometeorites (UCAMMs) have also been analysed for organic molecules. Meteorites have been thoroughly analysed for organic matter in the last few decades. However, the origin of the containing organic material (indigenous versus terrestrial contamination) is not certain (Brinton et al. 1998; Clemett et al. 1993, 1998; Flynn et al. 2003, 2004; Glavin et al. 2004; Keller et al. 2004; Matrajt et al. 2004, 2005). Furthermore, alteration that has occurred in the meteorite parent body(ies) (i.e., thermal metamorphism, aqueous alteration, impact shock and brecciation) may have influenced the chemical composition of chondrites (Browning et al. 1996; Palmer and Lauretta 2011; Vinogradoff et al. 2017), which are meteorites that have not been melted since formation, and constitute the majority (more than $80 \%$ ) of meteorites that reach the Earth surface. Carbonaceous chondrites are some of the most primitive chondrites as their bulk chemical composition is very similar to that of the sun (Lodders 2003). They consist of coarse ( $>10$ 's $\mu \mathrm{m}$ ) silicate components such as chondrules and calciumaluminium-rich inclusions (CAIs) set within a fine-grained $(<1 \mu \mathrm{m})$ matrix with dark appearance. Carbonaceous chondrites have high bulk meteoritic carbon content (up to $\sim 3.5$ weight total (wt)\%) (Alexander et al. 2007, 2017) present as silicon carbide, graphite, diamonds, carbonate and organic matter (Amari et al. 1990, 1993; Anders and Zinner 1993; Benedix et al. 2003; Fredriksson and Kerridge 1988; Grady et al. 1988; Hoppe et al. 1996; Lewis et al. 1987; Smith and Kaplan 1970; Zinner et al. 1995). Their organic content may be divided into two fractions: the solvent-soluble (SOM) one, which is obtained by extraction using different solvents, and the acid-insoluble (IOM) (Cody and Alexander 2005; Cody et al. 2002; Gardinier et al. 2000), which is obtained after demineralization (Robert and Epstein 1982).

The IOM is a dark, macromolecular organic solid which accounts for more than half of total organic carbon. Although the intact molecular structure of IOM is still unknown due to its complex configuration, determination of the macromolecular composition has been addressed by destructive techniques (e.g., pyrolysis, chemical degradation, and thermochemolysis) and non-destructive techniques (e.g. solid state-nuclear magnetic resonance (NMR), Fourier-transform infrared spectroscopy (FTIR), X-ray absorption near-edge structure (XANES), and electron paramagnetic resonance (EPR)) (See reviews by Sephton 2002; Pizzarello et al. 2006; Alexander et al. 2017; Glavin et al. 2018). Elemental compositions of IOM in the primitive meteorites are estimated as $\mathrm{C}_{100} \mathrm{H}_{58.8} \mathrm{~N}_{3.3} \mathrm{O}_{18.3}$ (Alexander et al. 2007). Pyrolysis of IOM yielded one to six ring-polycyclic aromatic hydrocarbons (PAHs) and O-, N-, S- containing aromatic compounds (e.g., Komiya et al. 1993; Sephton et al. 2004). Dichromic acid $\left(\mathrm{H}_{2} \mathrm{Cr}_{2} \mathrm{O}_{7}\right)$ oxidation (Hayatsu et al. 1977), alkaline copper oxide $(\mathrm{CuO})$ oxidation (Hayatsu et al. 1980), tetramethylammonium hydroxide (TMAH) thermochemolysis (Remusat et al. 2005), and ruthenium tetroxide $\left(\mathrm{RuO}_{4}\right)(\mathrm{Re}-$ musat et al. 2005) demonstrated that IOM contain short alkyl chains $\left(\mathrm{C}_{1}-\mathrm{C}_{9}\right)$. It has been suggested from solid state ${ }^{13} \mathrm{C}$ NMR studies that IOM in primitive carbonaceous chondrites is mainly constituted of polycyclic aromatic rings with aliphatic substituents (Cody et al. 
2002; Cronin et al. 1987; Gardinier et al. 2000). Cody et al. (2002) further concluded that IOM is composed of a small ring $\left(<\mathrm{C}_{20}\right)$ aromatic network $(\sim 60 \%)$ crosslinking with shortbranched aliphatic chains $(\sim 20 \%)$ and various oxygen $(\sim 20 \%)$ functional groups (e.g., ether, carbonyl groups). Variable enrichments in $\mathrm{D}$ and ${ }^{15} \mathrm{~N}$ from the IOM in the primitive carbonaceous chondrites have been reported (Alexander et al. 2007; Kerridge et al. 1987; Robert and Epstein 1982; Yang and Epstein 1983, 1984). Using nanoscale secondary ion mass spectrometry (NanoSIMS), it has been reported that D and ${ }^{15} \mathrm{~N}$ enrichments of IOM are located in submicron areas (Busemann et al. 2006) and they are often associated with nanoglobule textures (Nakamura-Messenger et al. 2006). Hydrous heating experiments of IOM indicated the presence of two fractions in the IOM, labile (enriched in deuterium D, ${ }^{13} \mathrm{C}$ and ${ }^{15} \mathrm{~N}$ ), and refractory (depleted in ${ }^{13} \mathrm{C}$ and ${ }^{15} \mathrm{~N}$ ) (Sephton et al. 2003, 2004).

There have been several hypotheses about the origin and synthetic mechanisms of IOM in the early solar system. It has been suggested that IOM is originated in extremely cold environments such as interstellar or solar nebula environments, which can explain enrichments of D and ${ }^{15} \mathrm{~N}$ in IOM in meteorites and IDPs (Alexander et al. 2007, 2010). On the other hand, contributions of Fischer-Tropsch (FTT) synthesis (e.g. Hill and Nuth 2003) and irradiation reaction of nebular gas (Kuga et al. 2015) have been also suggested for the formation of IOM. There is also another suggestion that the first IOM might have been formed via formose-type reaction of formaldehyde and ammonia on the meteorite parent body (Cody et al. 2011; Kebukawa et al. 2013).

The SOM content of meteorites has been extensively analysed and reviewed in the literature (e.g., Martins and Sephton 2009; Martins 2019), showing a high molecular diversity with tens of thousands of different molecular compositions (Schmitt-Kopplin et al. 2010). For more details (abundances, distribution and isotopic composition) regarding the specific SOM present in carbonaceous chondrites, including carboxylic acids, amino acids, diamino acids, dipeptides, diketopiperazines, sulfonic and phosphonic acids, purines, pyrimidines, sugars and sugar-related compounds, hydrocarbons, alcohols, amines, amides, aldehydes and ketones, please see Martins (2019). Their origin has been discussed against four main points: i) by detecting molecules that are not synthesised by terrestrial organisms; ii) by comparison to the organic content of samples collected from the terrestrial fallsite; iii) enantiomeric ratios for chiral molecules; and when that is not possible, and/or we have access to large quantities of sample, iv) measurement of the compound specific carbon/hydrogen/nitrogen isotopic compositions. This last item also points not only towards the source (terrestrial versus interstellar/parent body processing), but also towards the synthetic pathway of the organic compounds present in the SOM (Aléon and Robert 2004; Elsila et al. 2012; Millar et al. 1989; Robert 2003; Sandford et al. 2001; Terzieva and Herbst 2000; Tielens 1983; Yang and Epstein 1983). Indigenous soluble organic meteoritic compounds have been found to be enriched in $\mathrm{D},{ }^{13} \mathrm{C}$ and ${ }^{15} \mathrm{~N}$ (e.g. Cronin et al. 1993; Cooper et al. 1997; Elsila et al. 2012; Krishnamurthy et al. 1992; Yuen et al. 1984), with the enrichment in D and ${ }^{15} \mathrm{~N}$ of the SOM suggesting the synthesis of their precursors at cold temperatures, either in the interstellar medium or in the outer protoplanetary disk (Aléon and Robert 2004; Millar et al. 1989; Robert 2003; Sandford et al. 2001; Terzieva and Herbst 2000; Tielens 1983; Yang and Epstein 1983). However, one cannot exclude the possibility of extraterrestrial soluble organic compounds having low D, ${ }^{13} \mathrm{C}$ and ${ }^{15} \mathrm{~N}$. Details on the stable isotope measurements of each specific soluble organic compound present in carbonaceous chondrites have also been presented in detail in the literature (Aponte et al. 2011, 2014a, 2014b, 2015, 2016; Cooper et al. 1997, 2001; Cronin et al. 1993; Engel and Macko 1997; Ehrenfreund et al. 2001; Epstein et al. 1987; Gilmour and Pillinger 1994; Huang et al. 2005, 2015; Krishnamurthy et al. 1992; Martins 
2018; Martins et al. 2007b, 2008; Naraoka et al. 2000; Pizzarello and Huang 2002, 2005; Pizzarello and Yarnes 2016; Pizzarello et al. 1991, 2001, 2003, 2004, 2010; Yuen et al. 1984; for a review see Martins 2019).

\section{The Organic Matter Link Between Meteorites and Asteroids}

Ground- and space-based remote sensing measurements have linked the carbonaceous chondrites to asteroids with dark surfaces (i.e. low albedo). An increased awareness of this relationship first occurred in 1971 with the discovery that asteroid Bamberga has a very low albedo $(\sim 0.03)$ similar to the carbonaceous chondrites (Cruikshank and Kerridge 1992). It has since been established that dark, carbonaceous (C-type) asteroids comprise $\sim 70 \%$ of all known asteroids, in particular dominating the population in the cold, outer ( $>3 \mathrm{AU}$ ) regions of the main asteroid belt (e.g. DeMeo and Carry 2015). The relationship between some carbonaceous chondrites and C-type asteroids has been further strengthened by directly comparing telescope and spacecraft observations of asteroids with laboratory analyses of meteorites, and through a better understanding of space and terrestrial weathering effects (e.g. Cloutis et al. 2011a, 2011b; Cloutis et al. 2012a).

Most meteorite-asteroid comparisons focus on the visible to near infrared (VNIR, $\sim 0.5$ $4 \mu \mathrm{m})$ spectral region. Carbonaceous chondrites and C-type asteroids both have low overall reflectance and either neutral, slightly red (increasing reflectance with wavelength) or blue spectral slopes (decreasing reflectance with wavelength). Absorption features in this region are usually weak, with the most significant feature typically occurring at $\sim 0.7 \mu \mathrm{m}$ due to $\mathrm{Fe}^{2+}-\mathrm{Fe}^{3+}$ charge transfer, at $\sim 1 \mu \mathrm{m}$ from anhydrous silicates, and at $\sim 3 \mu \mathrm{m}$ from $-\mathrm{OH} / \mathrm{H}_{2} \mathrm{O}$ bound in hydrated minerals. The 3.3-3.6 $\mu \mathrm{m}$ region is diagnostic for organic materials as it contains absorptions from chemical groups such as $\mathrm{CH}$ and $\mathrm{NH}$. These features are regularly identified in laboratory spectra of bulk carbonaceous chondrites and extracted IOM and SOM, although again the absorptions are often weak due to the high opacity of the fine-grained samples, while terrestrial contamination of meteorites is always an issue (e.g. Kebukawa et al. 2009; Takir et al. 2013; Orthous-Daunay et al. 2013; Trigo-Rodríguez et al. 2014). It should also be noted that the surface-measured spectra of asteroids are influenced by the effects of space weathering, whereas with meteorites we are able to analyse sub-surface pieces, i.e., either the meteorite was fractured during atmospheric entry or was intentionally freshly fractured during its curation before being analysed, plus some meteoroids could be inner parts of asteroids and therefore not spatially weathered.

The spectral signatures of organic materials have only been observed for a small number of C-type asteroids, plus comets such as 67P/Churyumov-Gerasimenko (Capaccioni et al. 2015). Absorptions due to organics, which are present at only low levels in carbonaceous chondrites, are expected to be very weak in diffuse reflectance spectra from asteroids and therefore require high signal-to-noise remote measurements for their detection. The first report of organics on the surface of an asteroid was by Cruikshank and Brown (1987), who tentatively identified a feature at $3.4 \mu \mathrm{m}$, which they attributed to $\mathrm{C}-\mathrm{H}$, in the spectra of asteroid Elektra. From independent observations Campins et al. (2010) and Rivkin and Emery (2010) both suggested that the position and shape of features at $\sim 3.3 \mu \mathrm{m}$ and $\sim 3.4 \mu \mathrm{m}$ in spectra of asteroid Themis were consistent with the presence of aromatic and aliphatic $\mathrm{CH}_{2}$ and $\mathrm{CH}_{3}$ functional groups on the surface, respectively. Similarly, Licandro et al. (2011) reported that the reflectance spectra of asteroid Cybele are comparable to Themis and inferred the presence of complex organic materials from absorption features between 3.2-3.6 $\mu \mathrm{m}$. 
Fig. 1 The "3 $\mu \mathrm{m}$ " spectral region for Ceres compared to the hydrated carbonaceous chondrites Orgueil (CI1) and MacKay Glacier (MCY) 05231 (CM1). The Ceres spectrum is from De Sanctis et al. (2017) and the figure is taken from Bates et al. (2020)

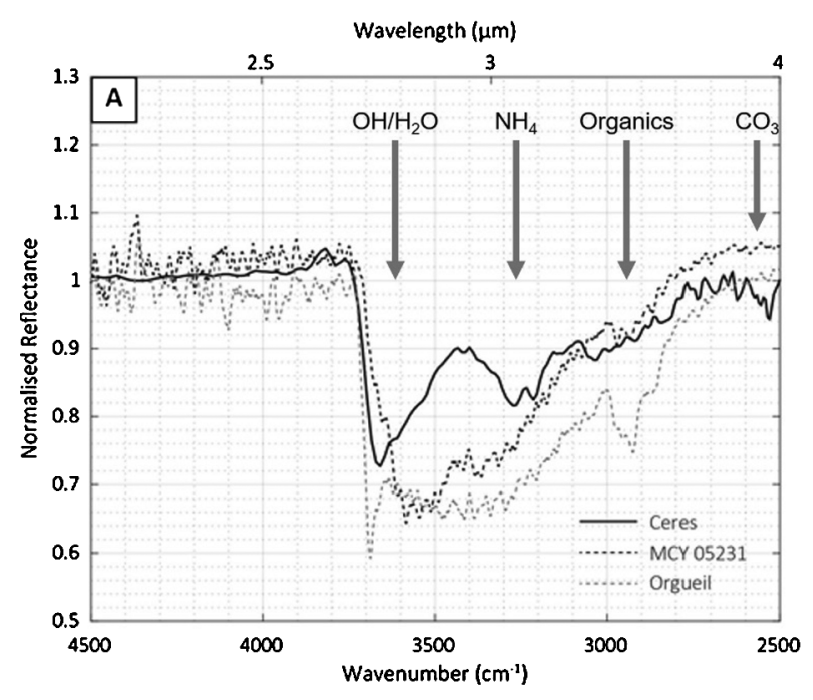

Ground-based observations have failed to unambiguously detect organic species on the surface of the dwarf planet Ceres, which is spectrally similar to the hydrated carbonaceous chondrites. However, in 2015 NASA's Dawn mission entered orbit around Ceres and characterised the surface in detail using a VNIR mapping spectrometer. Reflectance spectra collected by the Dawn spacecraft confirmed the presence of aliphatic organic compounds from $\mathrm{C}-\mathrm{H}$ vibrational absorptions at $\sim 3.4 \mu \mathrm{m}$ (De Sanctis et al. 2017; Fig. 1). Although it cannot be ruled out that the organics are widespread on the surface at low abundances, they are typically found concentrated in small, fresh ( $<50 \mathrm{Ma}$ ) impact craters (De Sanctis et al. 2017; Pieters et al. 2017). The depth of the $\sim 3.4 \mu \mathrm{m}$ band in these localised areas are significantly stronger than those found for meteoritic IOM, hinting at the presence of IOM-like organics at an abundance higher than that present in carbonaceous chondrites (De Sanctis et al. 2017; Kaplan et al. 2018). An important but currently unresolved question is whether the organics on Ceres come from an exogenous source (i.e. delivered by an organic-rich impactor) or result from an internal or near-surface endogenous process. Distinguishing between these two possibilities will have important implications for understanding the geophysical and geochemical history of Ceres and other carbonaceous bodies.

The Dawn mission ended in 2018 but there are currently two active space missions, JAXA's Hayabusa2 and NASA's OSIRIS-REx, characterising the carbonaceous near-Earth asteroids Ryugu and Bennu, which were selected due to their spectral links to the volatileand organic-rich carbonaceous chondrites. Preliminary results suggest that the surface of asteroid Ryugu contains a significant fraction of dehydrated minerals (Kitazato et al. 2019). Organic matter has not yet been detected on Ryugu but its surface is very dark (Watanabe et al. 2019; Sugita et al. 2019), possibly indicating a high abundance of carbon. Furthermore, a small lander of Hayabusa2 spacecraft, the Mobile Asteroid Surface Scout (MASCOT) observed a number of inclusions from the dark boulders on Ryugu, which are similar textures to those of less altered carbonaceous meteorites (Jaumann et al. 2019). MASCOT even revealed that Ryugu has low thermal conductivity and high porosity unlike any chondritic meteorites, while these thermal properties are similar to comets (Grott et al. 2019). In contrast, VNIR reflectance spectra from the surface of asteroid Bennu indicate an affinity with the hydrated carbonaceous chondrites (Hamilton et al. 2019). Again, organic materials have not been found but future higher-spatial-resolution spectral observations are expected to 
confirm their presence or absence on the surface of Bennu. A major aim for both Hayabusa2 and OSIRIS-REx is to collect samples from the surface of asteroids Ryugu and Bennu and return them to Earth (in 2020 for Hayabusa2, 2023 for OSIRIS-REx). Laboratory analyses of the returned samples will provide new insights into the formation and evolution of extraterrestrial organics and ground-truth remote sensing observations of solar system bodies.

\section{Organic Matter Comparison Between Meteorites and Dust Particles}

Several thousands of tons of extra-terrestrial matter fall on Earth, with meteorites representing the largest samples in terms of individual size. The major fraction in terms of total mass is nevertheless related to dust-among which, micrometeorites are recovered on the Earth's surface, and IDPs in the Earth's stratosphere. Chondrites are commonly considered as pieces of non-differentiated asteroids, although a cometary origin is discussed for some of them (Gounelle et al. 2006, 2008). The specific asteroidal class which chondrites are sampled from is not definitively established for each chondritic group. Ordinary chondrites are related to S-type asteroids; this was confirmed through the Hayabusa mission (Ebihara et al. 2011; Nagao et al. 2011; Nakamura et al. 2011; Noguchi et al. 2011; Tsuchiyama et al. 2011; Yurimoto et al. 2011). Moreover, type 1 and 2 carbonaceous chondrites are considered to be derived from C-type asteroids (e.g., Cloutis et al. 2011a, 2011b; Vernazza et al. 2015a). Nevertheless, the relative proportions of ordinary and carbonaceous chondrites is not similar to the relative abundances of the presumed asteroidal parent bodies (e.g., DeMeo and Carry 2013). Moreover, the limited number of chondrite groups does not reflect the large spectral variability observed among asteroids. On the other hand, dust can potentially be of asteroidal or cometary origin. There is a growing idea that some particles of dust might be samples from asteroidal parent bodies, which are not the parent bodies of meteorites. For example, some IDPs might be related to D- and P-type asteroids, most likely unrepresented among chondrites (Levison et al. 2009; Vernazza et al. 2015b).

Primitive meteorites and dust particles, which are preserved from severe heating resulting from terrestrial atmospheric entry, all contain a substantial fraction of organic compounds. Several reviews were published on organic matter in meteorites (e.g., Alexander et al. 2017; Gilmour 2014; Glavin et al. 2018). Some comparison of the organic matter in dust particles (IDPs and micrometeorites mostly collected in Antarctica) were previously published (Pizzarello et al. 2006; Quirico and Bonal 2019). The objective of this section is to summarise the key-points of the comparison on organic matter between chondrites and dust particles: mostly abundances, elemental and chemical compositions, and structure, as already stated in the literature. One of the big-picture questions the scientific community wants to address is related to the existence (or not) of several organic reservoir(s) in the solar protoplanetary disk potentially sampled by distinct parent bodies. Isotopic compositions of the organic matter appear to be key constraints. In this context, some new isotopic data acquired on Antarctic Micro-Meteorites (AMMs), completed by a review of the existing literature on IDPs and chondrites will also be discussed.

The chemical demineralization process, commonly applied to chondrites to isolate SOM and IOM, is highly challenging to apply on individual dust. So, most studies on dust are dealing with bulk analysis and not SOM or IOM analysis as for chondrites. Moreover, due to the small size and mass of individual dust, the analytical techniques that can be successfully applied are restricted in comparison to chondrites. Dust particles appear to be enriched in elemental carbon in comparison to chondrites. While carbonaceous chondrites contain only a few wt $\%$ of carbon, the bulk elemental abundance of carbon 
in IDPs can be as high as $45 \mathrm{wt} \%$ with a mean value around $15 \mathrm{wt} \%$ (Thomas et al. 1993), and in UCAMMs it can be up to the extreme abundance of $90 \mathrm{wt} \%$ (Dartois et al. 2013). At the micron scale, the $\mathrm{C} / \mathrm{H}$ ratios in bulk individual IDPs vary between 1 and 3 (Aléon et al. 2001), and between 2 and 6 in UCAMMs (Duprat et al. 2010). Emblematic chondrites are characterised in bulk by $\mathrm{C} / \mathrm{H}$ of $2.2-2.5,4.8$, and 1.9 in Orgueil (CI), Semarkona (UOC) and Murchison (CM), respectively (Alexander et al. 2012). The N/C ratios range from 0.03 to 0.24 in comet 81P/Wild dust particles (Cody et al. 2008; De Gregorio et al. 2011), from 0.05 to 0.15 in UCAMMs (Dartois et al. 2013; Yabuta et al. 2017), and from 0.03 to 0.04 in IOM primitive carbonaceous chondrites (Alexander et al. 2007). The $\mathrm{O} / \mathrm{C}$ ratios range from 0.11 to 0.67 in comet $81 \mathrm{P} /$ Wild dust particles (Cody et al. 2008) while they range from 0.10 to 0.22 in IOM primitive carbonaceous chondrites (Alexander et al. 2007). Nevertheless, it should be noted that distinct analytical techniques were used (e.g., NanoSIMS and SIMS vs. Elemental Analyzer). The ionization probability of a certain species being matrix dependent, the relationship between secondary ion yield of that species with its concentrations is not direct (e.g., Havette and Slodzian 1980). The determination of elemental ratios (potentially different matrix effects for distinct elements) is thus challenging by secondary ionization mass spectrometry.

Infrared micro-spectrometry was performed on IDPs (e.g., Flynn et al. 2003; Keller et al. 2004; Matrajt et al. 2005; Merouane et al. 2003; Muñoz Caro et al. 2006), AMMs (Battandier et al. 2018), UCAMMs (Dartois et al. 2013, 2018), and chondritic IOMs (e.g., Kebukawa et al. 2011; Orthous-Daunay et al. 2013). With the exception of UCAMMs, all these samples exhibit the spectral bands related to the $\mathrm{CH}_{2}$ and $\mathrm{CH}_{3}$ functions. The $\mathrm{CH}_{2} / \mathrm{CH}_{3}$ tend to be higher in dust particles in comparison to chondritic IOM (Quirico and Bonal 2019). However, $(i)$ the spectral acquisition and reduction were not always led in the same conditions (e.g., under vacuum or not); (ii) the $\mathrm{CH}_{2} / \mathrm{CH}_{3}$ ratio observed in dust could be modified through flash heating during atmospheric entry (e.g., discussed in Battandier et al. 2018); (iii) bulk measurements on dust might not be directly comparable to those on chondritic IOMs.

The structure of the polyaromatic matter present in the distinct families of primitive cosmomaterials was characterised by Raman micro-spectrometry by different research groups (chondrites: e.g., Bonal et al. 2006, 2007, 2016; Busemann et al. 2007; Quirico et al. 2003. AMMs: Dobrica et al. 2011; Battandier et al. 2018. IDPs: e.g., Wopenka 1988; Davidson et al. 2012; Starkey et al. 2013. Stardust particles: Sandford et al. 2006; Rotundi et al. 2008; Chan et al. 2019a). The experimental and analytical conditions are not always strictly similar, but the comparison stays informative. Dust collected in the stratosphere of the Earth or at the surface of the Earth obviously survived their atmospheric entry, but they have been heated to some degree, and thus preserve the pristine information to different degrees (Chan et al. 2019a). The consequences of atmospheric entry is particularly visible on the texture of MM and is used as a criterium of classification (Genge et al. 2008). Nevertheless, it was recently shown that even though their textures do not necessarily reveal heating through atmospheric entry, the organic matter of some fine-grained AMMs (Fg-AMMs) was in fact slightly modified (Battandier et al. 2018). Indeed, Fg-AMMs can either be made of hydrated or non-hydrated minerals, and non-hydrated ones tend to be characterised by a distinct structure of the polyaromatic organic (shift of some Raman spectral parameters) and a lower abundance of aliphatic organic matter in comparison to the hydrated ones. This was interpreted as resulting from heating through the atmospheric entry (Battandier et al. 2018). There is not an exact similitude in the spectral parameters describing the structure of the polyaromatic matter present in pristine dusts- the ones having best escaped atmospheric heating (Busemann et al. 2009; Dobrică et al. 2011; Davidson et al. 2012; 
Starkey et al. 2013; Battandier et al. 2018) and primitive chondrites (e.g., Bonal et al. 2016; Busemann et al. 2007; Quirico et al. 2018). This tends to underline the sampling of potentially distinct organic reservoirs, but dedicated experimental simulations are required to definitely rule out the effect of heating/oxidation and irradiation in space (Quirico and Bonal 2019).

The origin of isotopic enrichments observed in extra-terrestrial organics is still debated in the literature (solar and/or interstellar, see the review by Alexander et al. 2017). The precursor of organics in primitive cosmomaterials is considered by some authors as resulting from the assemblage of several components of interstellar and protoplanetary disk origins (e.g., Aléon 2010). The isotopic variability observed among cosmomaterials is then considered as reflecting variable abundance and preservation of the distinct components. On the other hand, large variations of $\mathrm{C}, \mathrm{H}$, and $\mathrm{N}$ isotopic compositions observed among chondritic IOMs have been interpreted by other authors as largely derived from secondary processes of a common precursor (e.g., Alexander et al. 1998, 2007). Isotopic anomalies may also imply that only a very small fraction of volatile interstellar matter survived the events of solar system formation, as an interstellar component with highly fractionated $\mathrm{H}$ and unfractionated $\mathrm{N}$ isotopes has been detected in ordinary chondrites (Aléon 2010). Isotopic heterogeneities at the micron- and submicron-scales are also observed within chondritic IOM (e.g., Busemann et al. 2006). Similar variations are observed between and within IDPs (Messenger 2000; Aléon et al. 2001; Messenger et al. 2003; Aléon et al. 2003; Keller et al. 2004; Floss et al. 2006; Busemann et al. 2009; Davidson et al. 2012; Starkey et al. 2014; Chan et al. 2019a). With the exception of UCAMMs (Duprat et al. 2010; Dartois et al. 2013; Yabuta et al. 2017), isotopic measurements in MM are very scarce, but bulk $\mathrm{C}$ and $\mathrm{N}$ isotopic measurements were recently performed in a series of Fg-AMMs (Battandier 2018) (Fig. 2). The $\mathrm{C}$ and $\mathrm{N}$ isotopic compositions of these Fg-AMMs are is highly variable in contrast to bulk compositions within individual group of chondrites (Kerridge 1985; Alexander et al. 2012; Floss et al. 2014). Moreover, there is no perfect match between individual Fg-AMMs and chondrite groups in terms of $\mathrm{C}$ and $\mathrm{N}$ isotopic compositions and there is no systematic variation in the bulk $\mathrm{C}$ or $\mathrm{N}$ isotopic compositions with the degree of hydration of the individual AMMs. Lastly, bulk chondrites are systematically ${ }^{13} \mathrm{C}$-poor, while dusts are roughly characterised by $-60<\delta^{13} \mathrm{C}(\% \circ)<+50$ (Fig. 2).

One could easily be tempted to interpret these differences between chondrites and dust in terms of distinct parent bodies, having sampled distinct organic reservoirs. However, some analytical details are worth being taken into consideration. It is interesting to note that among the measurements obtained by Battandier (2018) there seems to be a systematic difference between the measurements performed with two distinct instruments: Fg-AMMs characterised at Washington University (WU) appear to be systematically enriched in ${ }^{13} \mathrm{C}$ in comparison to those characterised at the Open University (OU), although no spectroscopic information allows to distinguish the considered samples in two groups. Interestingly IDPs characterised by Messenger (2000) are typically depleted in ${ }^{13} \mathrm{C}$ (only 2 out of 29 IDPs are ${ }^{13} \mathrm{C}$-rich), similar to those characterised by Starkey et al. (2014) (1 out of 10). In contrast, IDPs characterised by Floss et al. (2006) and Davidson et al. (2012) are ${ }^{13} \mathrm{C}$-enriched (only 3 out 29 and 1 out 10 are ${ }^{13} \mathrm{C}$-poor, respectively). Are these systematic ${ }^{13} \mathrm{C}$-enrichment or depletion related to specific series of samples, or to a potential uncontrolled analytical bias during individual measurement sessions? The measurements were performed following the established analytical methods in each of the laboratories. The standards were different: a mixture of synthetic $\mathrm{SiC}$ and $\mathrm{Si}_{3} \mathrm{~N}_{4}\left({ }^{14} \mathrm{~N} /{ }^{15} \mathrm{~N}=272\right)$ at WU vs. a HOBt polymer (hydroxybenzotriazole; $\mathrm{C}_{6} \mathrm{H}_{5} \mathrm{~N}_{3} \mathrm{O}$ ) at $\mathrm{OU}$, and although the e-Gun has never been used at WU, it was used for some measurements at OU. The limited size and mass of individual dust 


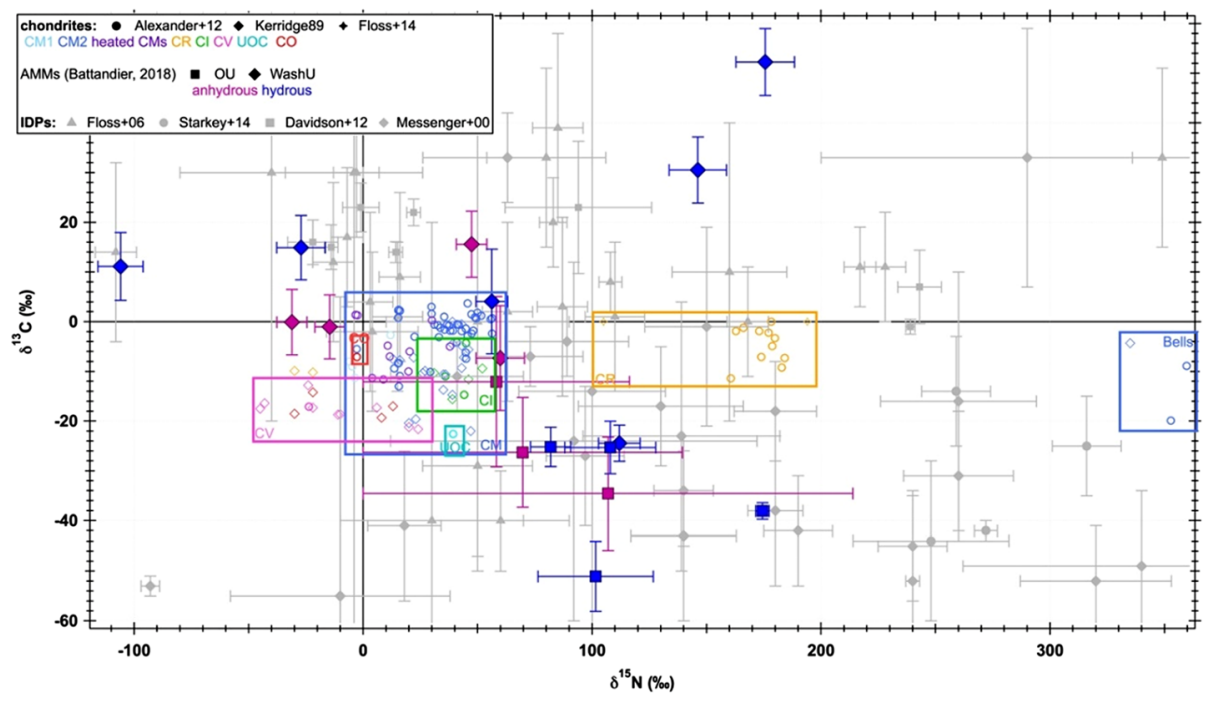

Fig. 2 Bulk $\mathrm{C}$ and $\mathrm{N}$ isotopic composition of Fg-AMMs, in comparison to bulk chondrites (open-colored symbols) and IDPs (grey symbols). Measurements on AMMs were performed with NanoSIMS at the Open University (square symbol) and at Washington University (diamond symbols) (Battandier 2018). Characterization by IR-spectroscopy of the Fg-AMMs: hydrated (in blue) or non-hydrated (in red) (Battandier et al. 2018). Data for chondrites and IDPs are from the literature, as cited in the text

clearly prevents repeat measurements using destructive techniques or using the same analytical technique as for chondrites (isotope-ratio mass spectrometry). Comparing the isotopic composition of dusts and chondrites, in particular $\mathrm{C}$, and interpreting the difference in terms of potential variability of organic reservoirs in the solar system might require performing systematic and multiple NanoSIMS measurements on chondrites. This analytical approach would allow $(i)$ comparing the isotopic compositions obtained independently by two distinct instruments; (ii) confirming the absence of analytical biases; (iii) confirming (or not) the large variability of the $\mathrm{C}$-isotopic composition of dusts in contrast to chondrites.

\section{In Which Type of Body We Expect to Find the Most Pristine Organic Material: On-Site Versus Sample Return}

Extensive worldwide meteorite collection has expanded year after year. Decades of meteoritic studies have improved our understanding of the organic distribution in our solar system. However, our meteorite record is biased as porous carbonaceous materials are underrepresented owing to their fragile nature that is prone to destruction during the atmospheric entry process. Also, most meteorite samples are finds with a long residence time on Earth (terrestrial ages for Antarctica finds rarely exceed $150 \mathrm{ka}$ (Welten et al. 2006), terrestrial ages in hot desert rarely exceed $50 \mathrm{ka}$ (Jull 2006), but in the Atacama desert the average terrestrial age is $710 \mathrm{ka}$ (Drouard et al. 2019)), and are therefore more likely to be contaminated by the fall site surroundings that can overprint any intrinsic organic signatures (Bada et al. 1998; Glavin et al. 1999; Martins et al. 2007a). Therefore, the elucidation of the main scientific problems related to the nature, distribution and evolution of organics necessitates the return of the most pristine extra-terrestrial samples to Earth. In addition, uncertainties remained 
in the source and geological information for the chemical evolution, which are not clearly recorded in the fallen extraterrestrial materials. In order to link the cosmochemical features of meteorites, IDPs, and AMMs with the geology of their original parent bodies, the exploration of small bodies is essential (Yabuta 2019).

\subsection{On-Site Versus Sample Return}

On-site space exploration can be conducted at reasonable costs and has provided us with insights into the organic inventory of our planetary and cometary neighbours (e.g., Freissinet et al. 2015; Goesmann et al. 2015; Klein 1978, 1979; Wright et al. 2015), demonstrating that organic compounds are ubiquitous in the solar system. In contrast, sample return missions (with the exception of Stardust so far) are often limited to closer inner solar system bodies (e.g. OSIRIS-REx, Hayabusa2, Hayabusa, Genesis). Unlike sample return missions, of which the returned samples are analysed in a terrestrial environment, on-site analysis of extra-terrestrial samples has a relatively lower risk of contamination. However, the samples can still be subjected to potential sources of contaminants that have bypassed the methodically controlled pre-flight, flight, and post-flight procedures, such as module engine exhaust, solvent, and chemical agents involved in wet chemistry experiments like organic extraction and derivatisation (Elsila et al. 2016; Freissinet et al. 2015) (further details regarding potential sources of terrestrial contamination is given in Chan et al. 2020, this issue). On-site exploration can consist of analytical instruments onboard spacecrafts or mobile vehicles, such as rovers that offer mobility and enables large-scale comparison of the organic properties to different surface features across the whole body.

However, despite our recent advancements in science and technology, the instrumentation required for high-precision and high-sensitivity characterisation of the organic composition cannot be easily miniaturised into flight compatible size. This downside of on-site exploration underscores the merit of a sample return mission. Any sample returned to Earth can be analysed by state-of-the-art instrumentation available in laboratories beyond the realm of on-site measurements by robotic spacecraft and processed by wet chemistry too complicated to be conducted without a dedicated laboratory facility. These instrumentations allow high-resolution imaging of the sample (e.g. synchrotron FTIR micro-spectroscopy, XANES spectroscopy, HRTEM and atomic force microscope infrared-spectroscopy (AFM-IR)) and isotopic analyses (e.g. NanoSIMS, gas chromatography combustion isotope ratio mass spectrometry (GC-C-IRMS)), both of which are crucial in order to elucidate the origins of the organics detected.

\subsection{Potential Future Sample Return Mission Target}

In order to detect some of the key compounds that play an important role in the origin of life on Earth and evolution of volatile components in the early solar system, it is imperative that we identify and return to Earth samples from a body that hosts abundant and pristine organic material, to be studied by the most precise and sensitive instrumentation available today, in order to answer more complex scientific questions such as where did organic symmetry breaking (i.e., prebiotic molecules with a slight enantiomeric excess) take place, and how did that influence the onset of life on Earth?

The significant proportion of hydrous minerals in carbonaceous chondrites suggests that their asteroid parent bodies have been processed by warm liquid water after accretion (Brearley 2006; Weisberg et al. 2006). Carbonaceous chondrites showing signs of 
aqueous alteration were also found to contain abundant organic material that include simple soluble organic precursors as well as complex and structurally diverse reaction products (Elsila et al. 2016; Glavin et al. 2010; Martins 2019; Martins et al. 2007b, 2015), therefore their parent bodies - C-, D- and P-type asteroids - are amongst the most scientifically exciting targets of sample return missions. In addition, L-enantiomeric excesses (Lee) of amino acids (e.g. the abiotic amino acid isovaline) were observed in meteorites that were aqueously altered, which indicates aqueous alteration as a plausible symmetry breaking mechanism (Glavin and Dworkin 2009; Pizzarello et al. 2003). Such nonracemic meteoritic amino acid composition is one of a few examples of molecular asymmetry present in the soluble fraction of carbonaceous meteorites, which offers important insights into the origin of terrestrial homochirality. Other examples include L-lactic acid in a diversity of carbonaceous meteorites (Pizzarello et al. 2010), sugar acids in several carbonaceous meteorites that contain large excesses of the D-enantiomer (Cooper and Rios 2016), and even IOM (Kawasaki et al. 2006). Different degrees of aqueous alteration processes are commonly associated with amino acid synthetic processes such as Strecker synthesis and Michael addition (Cronin and Chang 1993; Kebukawa et al. 2017; Peltzer and Bada 1978), although other more complex processes to synthesize amino acids may exist. It should be noted meteorites that have experienced a prolonged aqueous alteration history, such as the petrologic type 1 chondrites, have lower abundances of amino acids than meteorites that are mildly altered by water (Ehrenfreund et al. 2001; Martins et al. 2015). In fact, extensive aqueous alteration on the meteorite parent body may result in the decomposition of $\alpha$-amino acids (Botta et al. 2007; Martins et al. 2007b), as increasing lowtemperature chemical oxidation removes the aliphatic portions of the soluble and macromolecular organic material (Cody and Alexander 2005; Martins et al. 2006). Racemization of amino acids is particularly rapid in the presence of liquid (Bada and McDonald 1995; Bada et al. 1994; Cohen and Chyba 2000) that could erase signs of molecular asymmetry. Aqueously altered meteorites that were subsequently dehydrated, commonly referred to as thermally metamorphosed carbonaceous chondrites (TMCCs), have yet lower contents of soluble organic molecules (Shimoyama et al. 1989, 1990; Burton et al. 2014; Chan et al. 2016, 2018). Therefore, we should target a body which shows only a minor to mild degree of aqueous alteration due to its involvement in synthesis of organic material, the presence of ancient liquid water, and the role of aqueous processing in the origin of homochirality.

One of the most effective methods commonly used to identify such a primitive body is telescopic remote observation based on the spectrophotometric evidence of alteration minerals in their reflectance spectra in the wavelength range, as aqueous alteration in asteroids is evident by the absorption features in the $2.7-3.0 \mu \mathrm{m}$ region attributed to the presence of structural hydroxyl and adsorbed water, and thermal alteration evident by the absorption features indicative of high temperature anhydrous minerals (e.g. the absorption feature of olivine in the $0.8-1.3 \mu \mathrm{m}$ region) (Rivkin et al. 2002; Hiroi et al. 1996; Cloutis et al. 2012b). Hence, asteroids that show absorption features in both regions and have been altered aqueously and thermally would be less ideal. It is because the combined effect of both alteration regimes would have a profound destruction effect on the organics, and the complicated alteration history must be deciphered in order to appropriately address its influences on the organics. Indeed, the alteration event should be very mild and at low temperatures (e.g. aqueous alteration occurred at $<150^{\circ} \mathrm{C}$ on CM parent body (Zolensky et al. 1989; Verdier-Paoletti et al. 2016)). For instance, in the CM Paris meteorite, one of the most primitive carbonaceous chondrites, isovaline was found to be in racemic ratio (equal abundance 
between L- and D-isovaline), which indicates that the meteorite was only subjected to limited aqueous alteration, and therefore it likely reflects interstellar and/or presolar cloud organic inventory accreted prior to parent body processing (Martins et al. 2015).

Although meteorites with strong evidence of a high-temperature $\left(>300^{\circ} \mathrm{C}\right)$ thermal alteration history do not necessarily lack organic components (Bonal et al. 2006, 2007, 2016; Burton et al. 2012, 2014; Chan et al. 2012, 2016; Kitajima et al. 2002; Naraoka et al. 2004; Yabuta et al. 2005, 2010), the organic abundances are significantly lower in these meteorites. Their organic compounds are shown to be more structurally altered, and thus any pre-accretion information would have been modified alongside the heating processes. Hence, future sample return missions that aim at investigating primitive organics should avoid objects that show signs of significant thermal metamorphism. In addition, the aliphatic moieties are reduced by decarboxylation or conversion into aromatic $\mathrm{C}$ upon pyrolysis (e.g. Martins et al. 2006; Alexander et al. 2007; Chan et al. 2019a, 2019b). Amino acids can also be decomposed at temperatures as low as $100^{\circ} \mathrm{C}$ through processes such as decarboxylation, deamination and chain homolyses (e.g., Ratcliff et al. 1974; Bada and McDonald 1995; Pietrucci et al. 2018). Therefore, the amino acid abundances measured in meteorites that had experienced parent body metamorphism, including petrologic type 3 to 6 chondrites, are at least an order of magnitude lower than those in the mildly aqueously altered carbonaceous chondrites (Burton et al. 2012; Burton et al. 2014; Chan et al. 2012, 2016; Martins et al. 2007a). Parent body metamorphism may also modify the organic content by altering the relative abundances of different organic compounds, thus the detected organic composition does not represent the intrinsic population formed upon the asteroid accretion. For example, proteic amino acids are typically more thermodynamically unstable than nonproteic amino acids (e.g. $\beta$-alanine, $\gamma$-aminobutyric acid ( $\gamma$-ABA)) (Kitadai 2016), while hydrothermal alteration can produce simple volatile organic compounds such as amines, carboxylic acids and hydrocarbons at the expense of amino acids (Pietrucci et al. 2018). Therefore, the original organic compounds present in parent bodies that have experienced severe thermal metamorphism have been destroyed, i.e. their pre-accretion signature would be lost.

Data suggest that amino acids could be formed at low temperatures on the parent bodies of meteorites $\left(0\right.$ to $-10^{\circ} \mathrm{C}$ ) (Pizzarello and Groy 2011), which opens up new horizons for potential sample return missions from solar system objects that involve low temperature processes. Organic compounds may also be formed by solid-phase reactions on interstellar ice grains by energetic processing (e.g. cosmic rays and UV irradiation) (Augé et al. 2016, 2019). Laboratory analogues of the physical and chemical conditions of these interstellar ices (or pre-cometary icy grains) results in the formation of a residue that may contain glycerol, urea, glycolic acid (Nuevo et al. 2010), nucleobases (Nuevo et al. 2009, 2017), hydantoin (de Marcellus et al. 2011a), glyceraldehyde (de Marcellus et al. 2015), aldopentoses and sugar-related compounds (Meinert et al. 2016), and amino acids (Briggs et al. 1992; Kasamatsu et al. 1997; Bernstein et al. 2002; Muñoz Caro et al. 2002; Meinert and Meierhenrich 2012; de Marcellus et al. 2011b; Modica et al. 2014, 2018). Comets are also interesting targets as they are preserved cold - well below the typical liquid water phase temperatures - and thus have lower effects of thermal and aqueous processing, allowing the preservation of information on primitive grain aggregation and the initial inventory of pristine organics. Studies of comet 81P/Wild 2 samples show that the organics are more labile than the materials seen in meteorites, and enriched in heavy isotopes inherited from the interstellar medium (Brownlee et al. 2006; Sandford et al. 2006), therefore cometary organics offer a unique window into understanding the interstellar/protostellar organic inventory. Organic matter has also been detected in comet 67P/Churyumov-Gerasimenko. The Rosetta Orbiter Spectrometer for Ion 
and Neutral Analysis (ROSINA) and the Reflectron-type Time-Of-Flight mass spectrometer (RTOF) of the Rosetta mission determined the relative densities of the four major volatile species in the coma of the comet $\left(\mathrm{H}_{2} \mathrm{O}, \mathrm{CO}_{2}, \mathrm{CO}\right.$ and $\left.\mathrm{O}_{2}\right)$ and their seasonal evolution, showing that $\mathrm{CO}_{2}$ and $\mathrm{CO}$ were located on the surface of the southern hemisphere as a result of the strong erosion during the previous perihelion (Hoang et al. 2019; Combi et al. 2020). ROSINA also detected several hydrocarbons such as methane, ethane, ethylene, benzene and toluene, as well as CHO-bearing molecules (alcohols up to pentanol, aldehydes and acetone) (Altwegg et al. 2017). Several other classes of organic compounds were detected, e.g. CHN compounds (amines, hydrogen cyanide and acetonitrile), and CHNO (amides, isocyanic acid and glycine) (Altwegg et al. 2019). The presence of both glycine and methylamine in comet 67P suggests a possible synthesis pathway for amino acids without liquid water by the photochemistry of interstellar ices (methylamine and $\mathrm{CO}_{2}$ ) (Bossa et al. 2010).

Despite the availability of cometary samples such as the comet 81P/Wild 2 samples, UCAMMs and chondritic porous (CP) IDPs, they are of very limited total mass with only low amino acid and amines abundances (Elsila et al. 2009; Glavin et al. 2008). For example, the concentration of the amino acids in the Stardust-returned aerogel cells backing foil samples was very low and only glycine and $\varepsilon$-amino- $n$-caproic acid (EACA) were present at abundances barely sufficient for compound-specific carbon isotope measurements (Elsila et al. 2009). Although this implies the presence of amino acids derived from impacts of large cometary grains, their low abundances require large amounts of sample for their successful detection, which is rather difficult for sample return mission as samples are only available at a finite amount. Other than comets, icy objects such as the Saturnian and Jovian moons have also caught the attention as they were suggested to involve large-scale hydrovolcanism and have the potential of hosting a large water ocean beneath the icy crust (Carr et al. 1998; Postberg et al. 2008, 2009). The existence of organic molecules in the atmosphere of the Saturnian moon Enceladus account for a favourable environment suited for the formation of life's precursors and thus possibly host information on the origin of life (e.g., Khawaja et al. 2019; Postberg et al. 2008, 2009). As sample return missions aim to deliver the most pristine, unaltered samples from another celestial object to Earth, proper contamination control is imperative so as not to compromise the pristine nature of the extra-terrestrial sample (Chan et al. (2020) and Ferriere et al. (2020) - this issue).

\section{Conclusions}

The meteorite collections available all over the world have been a precious asset in the study of primitive organic matter. However, a precise conclusion is jeopardised if the samples are not carefully handled and curated. Alternatively, on-site and sample return space missions from asteroids and/or comets, while time-consuming, costly and complicated are fundamental towards obtaining pristine organic matter. While deciding between those two types of space missions (on-site versus sample return), one must consider the risk of contamination, and the available equipment. In both space missions, the indigenous organic content may encounter terrestrial organic molecules; even with excellent terrestrial curation facilities, returned samples will always be in contact with the terrestrial environment and therefore organic contaminants. In the case of on-site samples, the contamination may come from the module engine exhaust and chemical reagents present in wet chemistry experiments. Regarding the available equipment, sample return missions will certainly have access to more 
diverse and state-of-the-art instruments on Earth. Finally, the degree of alteration of the target body is a crucial factor to consider as it is necessary to have access to pristine and primitive samples. Target bodies should have minor to mild degrees of aqueous alteration, and no thermal metamorphism in order to increase the chances of acquiring data directly related to the accreted interstellar and/or presolar cloud organic inventory. Alternatively, the target bodies should have very low temperatures (below $0{ }^{\circ} \mathrm{C}$ ) in order to avoid alteration (aqueous and thermal) processes, e.g. space missions to comets. In summary, when planning future space missions, it is necessary to pay attention to all the above points. These considerations are fundamental to obtain extraterrestrial samples that will inform the planetary scientific community.

Acknowledgements Zita Martins was financed by FEDER - Fundo Europeu de Desenvolvimento Regional funds through the COMPETE 2020 - Operational Programme for Competitiveness and Internationalisation (POCI), and by Portuguese funds through FCT-Fundação para a Ciência e a Tecnologia in the framework of the project POCI-01-0145-FEDER-029932 (PTDC/FIS-AST/29932/2017). Centro de Química Estrutural acknowledges the financial support of Fundação para a Ciência e Tecnologia (UIDB/00100/2020). This research is framed within the College on Polar and Extreme Environments (Polar2E) of the University of Lisbon. Queenie Chan and Ashley King were supported by Science and Technology Facilities Council (STFC) grants \#ST/P000657/1 and \#ST/R000727/1, respectively. Lydie Bonal was supported by CNES through its "Système Solaire" program. Hikaru Yabuta was supported by JSPS KAKENHI Grant Numbers JP19H01954, JP18H04461 and by the Astrobiology Center Program of National Institutes of Natural Sciences (NINS) (Grant Number AB312007). The NanoSIMS measurements at OU have received funding from the European Union's Horizon 2020 research and innovation programme under grant agreement No 654208. Dr I. Franchi (OU) and Dr. F. Gyngard (WU) are thanked for their help with NanoSIMS measurements. We acknowledge the constructive comments from the Guest Editor and the anonymous Reviewers that improved the quality of this manuscript.

Publisher's Note Springer Nature remains neutral with regard to jurisdictional claims in published maps and institutional affiliations.

Open Access This article is licensed under a Creative Commons Attribution 4.0 International License, which permits use, sharing, adaptation, distribution and reproduction in any medium or format, as long as you give appropriate credit to the original author(s) and the source, provide a link to the Creative Commons licence, and indicate if changes were made. The images or other third party material in this article are included in the article's Creative Commons licence, unless indicated otherwise in a credit line to the material. If material is not included in the article's Creative Commons licence and your intended use is not permitted by statutory regulation or exceeds the permitted use, you will need to obtain permission directly from the copyright holder. To view a copy of this licence, visit http://creativecommons.org/licenses/by/4.0/.

\section{References}

J. Aléon, Multiple origins of nitrogen isotopic anomalies in meteorites and comets. Astrophys. J. 722, 1342$1351(2010)$

J. Aléon, F. Robert, Interstellar chemistry recorded by nitrogen isotopes in Solar System organic matter. Icarus 167, 424-430 (2004)

J. Aléon, C. Engrand, F. Robert et al., Clues to the origin of interplanetary dust particles from the isotopic study of their hydrogen-bearing phases. Geochim. Cosmochim. Acta 65, 4399-4412 (2001)

J. Aléon, F. Robert, M. Chaussidon, B. Marty, Nitrogen isotopic composition of macromolecular organic matter in interplanetary dust particles. Geochim. Cosmochim. Acta 67, 3773-3783 (2003)

C.M.O'D. Alexander, S.S. Russell, J.W. Arden et al., The origin of chondritic macromolecular organic matter: a carbon and nitrogen isotope study. Meteorit. Planet. Sci. 33, 603-622 (1998)

C.M.OD. Alexander, M. Fogel, H. Yabuta, G.D. Cody, The origin and evolution of chondrites recorded in the elemental and isotopic compositions of their macromolecular organic matter. Geochim. Cosmochim. Acta 71, 4380-4403 (2007)

C.M.O'D.Alexander, S.D. Newsome, M.L. Fogel, L.R. Nittler, H. Busemann, G.D. Cody, Deuterium enrichments in chondritic macromolecular material-implications for the origin and evolution of organics, water and asteroids. Geochim. Cosmochim. Acta 74, 4417-4437 (2010) 
C.M.O’D. Alexander, R. Bowden, M.L. Fogel et al., The provenances of asteroids, and their contributions to the volatile inventories of the terrestrial planets. Science 337, 721-723 (2012)

C.M.O'D. Alexander, G.D. Cody, B.T. De Gregorio et al., The nature, origin and modification of insoluble organic matter in chondrites, the major source of Earth's C and N. Chemie der Erde. Geochemistry 77, 227-256 (2017)

K. Altwegg, H. Balsiger, A. Bar-Nun et al., Prebiotic chemicals-amino acid and phosphorus-in the coma of comet 67P/Churyumov-Gerasimenko. Sci. Adv. 2, e1600285-e1600285 (2016)

K. Altwegg, H. Balsiger, J.J. Berthelier et al., Organics in comet 67P - a first comparative analysis of mass spectra from ROSINA-DFMS, COSAC and Ptolemy. Mon. Not. R. Astron. Soc. 469, S130 (2017)

K. Altwegg, H. Balsiger, S.A. Fuselier, Cometary chemistry and the origin of icy solar system bodies: the view after Rosetta. Annu. Rev. Astron. Astrophys. 57, 113-155 (2019)

S. Amari, A. Anders, A. Virag et al., Interstellar graphite in meteorites. Nature 345, 238-240 (1990)

S. Amari, P. Hoppe, E. Zinner et al., The isotopic compositions and stellar sources of meteoritic graphite grains. Nature 365, 806-809 (1993)

E. Anders, E. Zinner, Interstellar grains in primitive meteorites-diamond, silicon carbide, and graphite. Meteoritics 28, 490-514 (1993)

J.C. Aponte, M.R. Alexandre, Y. Wang et al., Effects of secondary alteration on the composition of free and IOM-derived monocarboxylic acids in carbonaceous chondrites. Geochim. Cosmochim. Acta 75, 2309-2323 (2011)

J.C. Aponte, R. Tarozo, M.R. Alexandre et al., Chirality of meteoritic free and IOM-derived monocarboxylic acids and implications for prebiotic organic synthesis. Geochim. Cosmochim. Acta 131, 1-12 (2014a)

J.C. Aponte, J.P. Dworkin, J.E. Elsila, Assessing the origins of aliphatic amines in the Murchison meteorite from their compound-specific carbon isotopic ratios and enantiomeric composition. Geochim. Cosmochim. Acta 141, 331-345 (2014b)

J.C. Aponte, J.P. Dworkin, J.E. Elsila, Indigenous aliphatic amines in the aqueously altered Orgueil meteorite. Meteorit. Planet. Sci. 50, 1733-1749 (2015)

J.C. Aponte, H.L. McLain, J.P. Dworkin et al., Aliphatic amines in Antarctic CR2, CM2, and CM1/2 carbonaceous chondrites. Geochim. Cosmochim. Acta 189, 296-311 (2016)

B. Augé, E. Dartois, C. Engrand et al., Irradiation of nitrogen-rich ices by Swift heavy ions. Clues for the formation of ultracarbonaceous micrometeorites. Astron. Astrophys. 592, A99 (2016)

B. Augé, E. Dartois, J. Duprat et al., Hydrogen isotopic anomalies in extraterrestrial organic matter: role of cosmic ray irradiation and implications for UCAMMs. Astron. Astrophys. 627, A122 (2019)

J.L. Bada, G.D. McDonald, Amino acid racemization on Mars: implications for the preservation of biomolecules from an extinct martian biota. Icarus 114, 139-143 (1995)

J.L. Bada, X.S. Wang, H.N. Poinar et al., Amino acid racemization in amber-entombed insects: implications for DNA preservation. Geochim. Cosmochim. Acta 58, 3131-3135 (1994)

J.L. Bada, D.P. Glavin, G.D. McDonald, L. Becker, A search for endogenous amino acids in martian meteorite ALH84001. Science 279, 362-365 (1998)

H.C. Bates, A.J. King, K.L. Donaldson Hanna et al., Linking mineralogy and spectroscopy of highly aqueously altered CM and CI carbonaceous chondrites in preparation for primitive asteroid sample return. Meteorit. Planet. Sci. 55, 77-101 (2020)

M. Battandier, Etude d'une série de micrométéorites antarctiques: caractérisation multi-analytique et comparaison à des chondrites carbonées. Doctoral dissertation from, Université Grenoble Alpes (2018)

M. Battandier, L. Bonal, E. Quirico, P. Beck, C. Engrand, J. Duprat, E. Dartois, Characterization of the organic matter and hydration state of Antarctic micrometeorites: a reservoir distinct from carbonaceous chondrites. Icarus 306, 74-93 (2018)

G.K. Benedix, L.A. Leshin, J. Farquhar et al., Carbonates in CM2 chondrites: constraints on alteration conditions from oxygen isotopic compositions and petrographic observations. Geochim. Cosmochim. Acta 67, 1577-1588 (2003)

M.P. Bernstein, J.P. Dworkin, S.A. Sandford et al., Racemic amino acids from the ultraviolet photolysis of interstellar ice analogues. Nature 416, 401-403 (2002)

D. Bockelée-Morvan et al., AMBITION-Comet Nucleus Cryogenic Sample Return. A white paper for ESA Voyage 2050 long-term plan (2019). https://arxiv.org/pdf/1907.11081.pdf

D. Bockelée-Morvan, J. Crovisier, M.J. Mumma et al., The composition of cometary volatiles, in Comets II, ed. by M. Festou, H.U. Keller, H.A. Weaver (University of Arizona Press, Tucson, 2004), pp. 391-423

L. Bonal, E. Quirico, M. Bourot-Denise et al., Determination of the petrologic type of CV3 chondrites by Raman spectroscopy of included organic matter. Geochim. Cosmochim. Acta 70, 1849-1863 (2006)

L. Bonal, M. Bourot-Denise, E. Quirico et al., Organic matter and metamorphic history of CO chondrites. Geochim. Cosmochim. Acta 71, 1605-1623 (2007)

L. Bonal, E. Quirico, L. Flandinet et al., Thermal history of type 3 chondrites from the Antarctic meteorite collection determined by Raman spectroscopy of their polyaromatic carbonaceous matter. Geochim. Cosmochim. Acta 189, 312-337 (2016) 
J.B. Bossa, F. Borget, F. Duvernay et al., How a usual carbamate can become an unusual intermediate: a new chemical pathway to form glycinate in the interstellar medium. J. Phys. Org. Chem. 23, 333-339 (2010)

O. Botta, Z. Martins, P. Ehrenfreund, Amino acids in Antarctic CM1 meteorites and their relationship to other carbonaceous chondrites. Meteorit. Planet. Sci. 42, 81-92 (2007)

A.J. Brearley, The Action of Water (The University of Arizona Press, Tucson, 2006), pp. 584-624

R. Briggs, G. Ertem, J.P. Ferris et al., Comet Halley as an aggregate of interstellar dust and further evidence for the photochemical formation of organics in the interstellar medium. Orig. Life Evol. Biosph. 22, 287-307 (1992)

K.L.F. Brinton, C. Engrand, D.P. Glavin et al., A search for extraterrestrial amino acids in carbonaceous Antarctic micrometeorites. Orig. Life Evol. Biosph. 28, 413-424 (1998)

L.B. Browning, H.Y. McSween, M.E. Zolensky, Correlated alteration effects in CM carbonaceous chondrites. Geochim. Cosmochim. Acta 60, 2621-2633 (1996)

D. Brownlee, P. Tsou, J. Aléon et al., Comet 81P/Wild 2 under a microscope. Science 314, 1711-1716 (2006)

A.S. Burton, J.E. Elsila, M.P. Callahan et al., A propensity for $n-\omega$-amino acids in thermally altered Antarctic meteorites. Meteorit. Planet. Sci. 47, 374-386 (2012)

A.S. Burton, S. Grunsfeld, J.E. Elsila et al., The effects of parent-body hydrothermal heating on amino acid abundances in CI-like chondrites. Polar Sci. 8, 255-263 (2014)

H. Busemann, A.F. Young, C.M.O'D. Alexander et al., Interstellar chemistry recorded in organic matter from primitive meteorites. Science 312, 727-730 (2006)

H. Busemann, C.M.OD. Alexander, L.R. Nittler, Characterization of insoluble organic matter in primitive meteorites by micro-Raman spectroscopy. Meteorit. Planet. Sci. 42, 1387-1416 (2007)

H. Busemann, A.N. Nguyen, G.D. Cody et al., Ultra-primitive interplanetary dust particles from the comet 26P/Grigg-Skjellerup dust stream collection. Earth Planet. Sci. Lett. 288, 44-57 (2009)

H. Campins, K. Hargrove, N. Pinilla-Alonso et al., Water ice and organics on the surface of the asteroid 24 Themis. Nature 464, 1320-1321 (2010)

F. Capaccioni et al., The organic-rich surface of comet 67P/Churyumov-Gerasimenko as seen by VIRTIS/Rosetta. Science 347, aaa0628 (2015)

M.H. Carr, M.J.S. Belton, C.R. Chapman, Evidence for a subsurface ocean on Europa. Nature 391, 363 (1998)

H.-S. Chan, Z. Martins, M.A. Sephton, Amino acid analyses of type 3 chondrites Colony, Ornans, Chainpur, and Bishunpur. Meteorit. Planet. Sci. 47, 1502-1516 (2012)

Q.H.S. Chan, Y. Chikaraishi, Y. Takano et al., Amino acid compositions in heated carbonaceous chondrites and their compound-specific nitrogen isotopic ratios. Earth Planets Space 68, 7 (2016)

Q.H.S. Chan, M. Zolensky, Y. Kebukawa et al., Organic matter in extraterrestrial water-bearing salt crystals. Sci. Adv. 4, eaao3521 (2018)

Q.H.S. Chan et al., Organics preserved in anhydrous interplanetary dust particles: pristine or not? Meteorit. Planet. Sci. (2019a). https://doi.org/10.1111/maps.13414.

Q.H.S. Chan, A. Nakato, Y. Kebukawa et al., Heating experiments of the Tagish Lake meteorite: investigation of the effects of short-term heating on chondritic organics. Meteorit. Planet. Sci. 54, 104-125 (2019b)

Q.H.S. Chan, R. Stroud, Z. Martins, H. Yabuta, Concerns of organic contamination for sample return space missions. Space Sci. Rev. (2020). https://doi.org/10.1007/s11214-020-00678-7

S.J. Clemett, C.R. Maechling, R.N. Zare et al., Identification of complex aromatic molecules in individual interplanatery dust particles. Science 262, 721-725 (1993)

S.J. Clemett, X.D.F. Chillier, S. Gillette et al., Observation of indigenous polycyclic aromatic hydrocarbons in 'giant' carbonaceous Antarctic micrometeorites. Orig. Life Evol. Biosph. 28, 425-448 (1998)

E.A. Cloutis, T. Hiroi, M.J. Gaffey et al., Spectral reflectance properties of carbonaceous chondrites: 1 . CI chondrites. Icarus 212, 180-209 (2011a)

E.A. Cloutis, P. Hudon, T. Hiroi et al., Spectral reflectance properties of carbonaceous chondrites: 2. CM chondrites. Icarus 216, 309-346 (2011b)

E.A. Cloutis, P. Hudon, T. Hiroi et al., Spectral reflectance properties of carbonaceous chondrites: 6. CV chondrites. Icarus 221, 328-358 (2012a)

E.A. Cloutis et al., Spectral reflectance properties of carbonaceous chondrites 4: aqueously altered and thermally metamorphosed meteorites. Icarus 220, 586-617 (2012b)

G.D. Cody, C.M.OD. Alexander, NMR studies of chemical structural variation of insoluble organic matter from different carbonaceous chondrites groups. Geochim. Cosmochim. Acta 69, 1085-1097 (2005)

G.D. Cody, C.M.OD. Alexander, F. Tera, Solid-state (1H and 13C) nuclear magnetic resonance spectroscopy of insoluble organic residue in the Murchison meteorite: a self-consistent quantitative analysis. Geochim. Cosmochim. Acta 66, 1851-1865 (2002)

G.D. Cody, H. Ade, C.M.OD. Alexander et al., Quantitative organic and light-element analysis of comet 81P/Wild 2 particles using C-, N-, and O- $\mu$-XANES. Meteorit. Planet. Sci. 43, 353-365 (2008) 
G.D. Cody, E. Heying, C.M.OD. Alexander et al., Establishing a molecular relationship between chondritic and cometary organic solids. Proc. Natl. Acad. Sci. USA 108, 19171-19176 (2011)

B.A. Cohen, C.F. Chyba, Racemization of meteoritic amino acids. Icarus 145, 272-281 (2000)

M. Combi, Y. Shou, N. Fougere et al., The surface distributions of the production of the major volatile species, $\mathrm{H} 2 \mathrm{O}, \mathrm{CO} 2, \mathrm{CO}$ and $\mathrm{O} 2$, from the nucleus of comet 67P/Churyumov-Gerasimenko throughout the Rosetta Mission as measured by the ROSINA double focusing mass spectrometer. Icarus 335, $113421(2020)$

G. Cooper, A. Rios, Enantiomer excesses of rare and common sugar derivatives in carbonaceous meteorites. Proc. Natl. Acad. Sci. USA 113, E3322-E3331 (2016)

G.W. Cooper, M.H. Thiemens, T.L. Jackson et al., Sulfur and hydrogen isotope anomalies in meteorite sulfonic acids. Science 277, 1072-1074 (1997)

G. Cooper, N. Kimmich, W. Belisle et al., Carbonaceous meteorites as a source of sugar-related organic compounds for the early Earth. Nature 414, 879-883 (2001)

J.R. Cronin, S. Chang, Organic matter in meteorites: molecular and isotopic analyses of the Murchison meteorite, in The Chemistry of Life's Origins, ed. by J.M. Greenberg, C.X. Mendoza-Gómez, V. Pirronello. NATO ASI Series, vol. 416 (Kluwer Academic Publishers, Norwell, 1993), pp. 209-258

J.R. Cronin, S. Pizzarello, J.S. Frye, ${ }^{13}$ C NMR spectroscopy of the insoluble carbon of carbonaceous chondrites. Geochim. Cosmochim. Acta 51, 299-303 (1987)

J.R. Cronin, S. Pizzarello, S. Epstein et al., Molecular and isotopic analyses of the hydroxy acids, dicarboxylic acids, and hydroxydicarboxylic acids of the Murchison meteorite. Geochim. Cosmochim. Acta 57, 4745-4752 (1993)

J. Crovisier, D. Bockelée-Morvan, Remote observations of the composition of cometary volatiles. Space Sci. Rev. 90, 19-32 (1999)

J. Crovisier, N. Biver, D. Bockelee-Morvan et al., The chemical diversity of comets. Earth Moon Planets 105, 267-272 (2009)

D.P. Cruikshank, R.H. Brown, Organic matter on asteroid 130 Elektra. Science 238, 183-184 (1987)

D.P. Cruikshank, J.F. Kerridge, Organic material: asteroids, meteorites, and planetary satellites, in Exobiology in Solar System Exploration (1992), pp. 159-176

E. Dartois, C. Engrand, R. Brunetto et al., UltraCarbonaceous Antarctic micrometeorites, probing the Solar System beyond the nitrogen snow-line. Icarus 224, 243-252 (2013)

E. Dartois, C. Engrand, J. Duprat et al., Dome C ultracarbonaceous Antarctic micrometeorites. Infrared and Raman fingerprints. Astron. Astrophys. 609, A65 (2018)

J. Davidson, H. Busemann, I.A. Franchi, A NanoSIMS and Raman spectroscopic comparison of interplanetary dust particles from comet Grigg-Skjellerup and non-Grigg Skjellerup collections. Meteorit. Planet. Sci. 47, 1748-1771 (2012)

B.T. De Gregorio, R.M. Stroud, G.D. Cody et al., Correlated microanalysis of cometary organic grains returned by Stardust. Meteorit. Planet. Sci. 46, 1376-1396 (2011)

P. de Marcellus, M. Bertrand, M. Nuevo et al., Prebiotic significance of extraterrestrial ice photochemistry: detection of hydantoin in organic residues. Astrobiology 11, 847-854 (2011a)

P. de Marcellus, C. Meinert, M. Nuevo et al., Non-racemic amino acid production by ultraviolet irradiation of achiral interstellar ice analogs with circularly polarized light. Astrophys. J. Lett. 727, 1-6 (2011b)

P. de Marcellus, C. Meinert, I. Myrgorodska et al., Aldehydes and sugars from evolved precometary ice analogs: importance of ices in astrochemical and prebiotic evolution. Proc. Natl. Acad. Sci. USA 112, 965-970 (2015)

M.C. De Sanctis, E. Ammannito, H.Y. McSween et al., Localized aliphatic organic material on the surface of Ceres. Science 355, 719-722 (2017)

F.E. DeMeo, B. Carry, The taxonomic distribution of asteroids from multi-filter all-sky photometric surveys. Icarus 226, 723-741 (2013)

F.E. DeMeo, B. Carry, Solar System evolution from compositional mapping of the asteroid belt. Nature 505, 629-634 (2015)

E. Dobrică, C. Engrand, E. Quirico et al., Raman characterization of carbonaceous matter in CONCORDIA Antarctic micrometeorites. Meteorit. Planet. Sci. 46, 1363-1375 (2011)

A. Drouard, J. Gattacceca, A. Hutzler, P. Rochette, R. Braucher, D. Bourlès, M. Gounelle, A. Morbidelli, V. Debaille, M. Van Ginneken, M. Valenzuela, Y. Quesnel, R. Martinez, The meteorite flux of the past 2 m.y. recorded in the Atacama Desert. Geology 47, 673-676 (2019)

J. Duprat, E. Dobrica, C. Engrand et al., Extreme deuterium excesses in ultracarbonaceous micrometeorites from Central Antarctic snow. Science 328, 742-745 (2010)

M. Ebihara, S. Sekimoto, N. Shirai et al., Neutron activation analysis of a particle returned from asteroid Itokawa. Science 333, 1119-1121 (2011)

P. Ehrenfreund, D.P. Glavin, O. Botta et al., Extraterrestrial amino acids in Orgueil and Ivuna: tracing the parent body of CI type carbonaceous chondrites. Proc. Natl. Acad. Sci. USA 98, 2138-2141 (2001) 
J.E. Elsila, D.P. Glavin, J.P. Dworkin, Cometary glycine detected in samples returned by Stardust. Meteorit. Planet. Sci. 44, 1323-1330 (2009)

J.E. Elsila, S.B. Charnley, A.S. Burton et al., Compound-specific carbon, nitrogen, and hydrogen isotopic ratios for amino acids in $\mathrm{CM}$ and $\mathrm{CR}$ chondrites and their use in evaluating potential formation pathways. Meteorit. Planet. Sci. 47, 1517-1536 (2012)

J.E. Elsila, J.C. Aponte, D.G. Blackmond et al., Meteoritic amino acids: diversity in compositions reflects parent body histories. ACS Central Science 2, 370-379 (2016)

M.H. Engel, S.A. Macko, Isotopic evidence for extraterrestrial non-racemic amino acids in the Murchison meteorite. Nature 389, 265-268 (1997)

S. Epstein, R.V. Krishnamurthy, J.R. Cronin et al., Unusual stable isotope ratios in amino acid and carboxylic acid extracts from the Murchison meteorite. Nature 326, 477-479 (1987)

C. Floss, F.J. Stadermann, J.P. Bradley et al., Identification of isotopically primitive interplanetary dust particles: a NanoSIMS isotopic imaging study. Geochim. Cosmochim. Acta 70, 2371-2399 (2006)

C. Floss, C. Le Guillou, A. Brearley, Coordinated NanoSIMS and FIB-TEM analyses of organic matter and associated matrix materials in CR3 chondrites. Geochim. Cosmochim. Acta 139, 1-25 (2014)

G.J. Flynn, L.P. Keller, M. Feser et al., The origin of organic matter in the solar system: evidence from the interplanetary dust particles. Geochim. Cosmochim. Acta 67, 4791-4806 (2003)

G.J. Flynn, L.P. Keller, C. Jacobsen et al., An assessment of the amount and types of organic matter contributed to the Earth by interplanetary dust. Adv. Space Res. 33, 57-66 (2004)

K. Fredriksson, J.F. Kerridge, Carbonates and sulfates in CI chondrites-formation by aqueous activity on the parent body. Meteoritics 23, 35-44 (1988)

C. Freissinet, D.P. Glavin, P.R. Mahaffy et al., Organic molecules in the Sheepbed Mudstone, Gale Crater, Mars. J. Geophys. Res., Planets 120, 495-514 (2015)

A. Gardinier, S. Derenne, F. Robert et al., Solid state CP/MAS 13C NMR of the insoluble organic matter of the Orgueil and Murchison meteorites: quantitative study. Earth Planet. Sci. Lett. 184, 9-21 (2000)

M.J. Genge, C. Engrand, M. Gounelle et al., The classification of micrometeorites. Meteorit. Planet. Sci. 43, 497-515 (2008)

I. Gilmour, Structural and isotopic analysis of organic matter in carbonaceous chondrites, in Reference Module in Earth Systems and Environmental Sciences-Treatise on Geochemistry 1 (2014), pp. 215-233

I. Gilmour, C.T. Pillinger, Isotopic compositions of individual polycyclic aromatic hydrocarbons from the Murchison meteorite. Mon. Not. R. Astron. Soc. 269, 235-240 (1994)

D.P. Glavin, J. Dworkin, Enrichment of the amino acid L-isovaline by aqueous alteration on CI and CM meteorite parent bodies. Proc. Natl. Acad. Sci. USA 106, 5487-5492 (2009)

D.P. Glavin, J.L. Bada, K.L.F. Brinton et al., Amino acids in the Martian meteorite Nakhla. Proc. Natl. Acad. Sci. 96, 8835-8838 (1999)

D.P. Glavin, G. Matrajt, J.L. Bada, Re-examination of amino acids in Antarctic micrometeorites. Adv. Space Res. 33, 106-113 (2004)

D.P. Glavin, J.P. Dworkin, S.A. Sandford, Detection of cometary amines in samples returned by Stardust. Meteorit. Planet. Sci. 43, 399-413 (2008)

D.P. Glavin, M.P. Callahan, J.P. Dworkin et al., The effects of parent body processes on amino acids in carbonaceous chondrites. Meteorit. Planet. Sci. 45, 1948-1972 (2010)

D.P. Glavin, C.M.O'D. Alexander, J.C. Aponte, J.P. Dworkin, J.E. Elsila, H. Yabuta, The origin and evolution of organic matter in carbonaceous chondrites and links to their parent bodies, in Primitive Meteorites and Asteroids Physical, Chemical and Spectroscopic Observations Paving the Way to Exploration (2018), pp. 205-271

F. Goesmann, H. Rosenbauer, J.H. Bredehöft et al., Organic compounds on comet 67P/ChuryumovGerasimenko revealed by COSAC mass spectrometry. Science 349, aab0689 (2015)

M.A. Gounelle, P. Spurný, P.A. Bland, The orbit and atmospheric trajectory of the Orgueil meteorite from historical records. Meteorit. Planet. Sci. 41, 135-150 (2006)

M. Gounelle, A. Morbidelli, P.A. Bland et al., Meteorites from the outer solar system?, in The Solar System Beyond Neptune, ed. by M.A. Barucci, H. Boehnhardt, D.P. Cruikshank, A. Morbidelli (University of Arizona Press, Tucson, 2008), pp. 525-541

M.M. Grady, I.P. Wright, P.K. Swart et al., The carbon and oxygen isotopic composition of meteoritic carbonates. Geochim. Cosmochim. Acta 52, 2855-2866 (1988)

M. Grott, J. Knollenberg, M. Hamm, Low thermal conductivity boulder with high porosity identified on C-type asteroid (162173) Ryugu. Nat. Astron. 3, 971-976 (2019)

V.E. Hamilton, A.A. Simon, P.R. Christensen et al., Evidence for widespread hydrated minerals on asteroid (101955) Bennu. Nat. Astron. 3, 332-340 (2019)

A. Havette, G. Slodzian, Matrix effects in secondary ion emission: quantitative analysis of silicates. J. Phys. Lett. 41, L247-L250 (1980) 
R. Hayatsu, S. Matsuoka, E. Anders et al., Origin of organic matter in the early solar system. VII. The organic polymer in carbonaceous chondrites. Geochim. Cosmochim. Acta 41, 1325-1339 (1977)

R. Hayatsu, R.E. Winans, R.G. Scott et al., Phenolic ethers in the organic polymer of the Murchison meteorite. Science 207, 1202-1204 (1980)

H.G.M. Hill, J.A. Nuth, The catalytic potential of cosmic dust: implications for prebiotic chemistry in the solar nebula and other protoplanetary systems. Astrobiology 3, 291-304 (2003)

T. Hiroi et al., Thermal metamorphism of the C, G, B, and F asteroids seen from the $0.7 \mu \mathrm{m}, 3 \mu \mathrm{m}$, and UV absorption strengths in comparison with carbonaceous chondrites. Meteorit. Planet. Sci. 31, 321-327 (1996)

M. Hoang, P. Garnier, H. Gourlaouen et al., Two years with comet 67P/Churyumov-Gerasimenko: H2O, CO2, and CO as seen by the ROSINA/RTOF instrument of Rosetta. Astron. Astrophys. 630, A33 (2019)

P. Hoppe, R. Strebel, P. Eberhardt et al., Type II supernova matter in a silicon carbide grain from the Murchison meteorite. Science 272, 1314-1316 (1996)

Y. Huang, Y. Wang, M.R. Alexandre et al., Molecular and compound-specific isotopic characterization of monocarboxylic acids in carbonaceous meteorites. Geochim. Cosmochim. Acta 69, 1073-1084 (2005)

Y. Huang, J.C. Aponte, J. Zhao et al., Hydrogen and carbon isotopic ratios of polycyclic aromatic compounds in two CM2 carbonaceous chondrites and implications for prebiotic organic synthesis. Earth Planet. Sci. Lett. 426, 101-108 (2015)

R. Jaumann, N. Schmitz, T.-M. Ho et al., Images from the surface of asteroid Ryugu show rocks similar to carbonaceous chondrite meteorites. Science 365, 817-820 (2019)

A.J.T. Jull, Terrestrial Ages of Meteorites, in Meteorites and the Early Solar System II, ed. by D.S. Lauretta, H.Y. McSween Jr. (University of Arizona Press, Tucson, 2006), pp. 889-905. 943 pp.

H.H. Kaplan, R.E. Milliken, C.M.OD. Alexander, New constraints on the abundance and composition of organic matter on Ceres. Geophys. Res. Lett. 45, 5274-5282 (2018)

T. Kasamatsu, T. Kaneko, T. Saito et al., Formation of organic compounds in simulated interstellar media with high energy particles. Bull. Chem. Soc. Jpn. 70, 1021 (1997)

T. Kawasaki, K. Hatase, Y. Fujii et al., The distribution of chiral asymmetry in meteorites: an investigation using asymmetric autocatalytic chiral sensors. Geochim. Cosmochim. Acta 70, 5395-5402 (2006)

Y. Kebukawa, S. Nakashima, T. Otsuka et al., Rapid contamination during storage of carbonaceous chondrites prepared for micro FTIR measurements. Meteorit. Planet. Sci. 44, 545-557 (2009)

Y. Kebukawa, C.M.OD. Alexander, G.D. Cody, Compositional diversity in insoluble organic matter in type 1,2 and 3 chondrites as detected by infrared spectroscopy. Geochim. Cosmochim. Acta 75, 3530-3541 (2011)

Y. Kebukawa, A.L.D. Kilcoyne, G.D. Cody, Exploring the potential formation of organic solids in chondrites and comets through polymerization of interstellar formaldehyde. Astrophys. J. 771, 19 (2013)

Y. Kebukawa, Q.H.S. Chan, S. Tachibana, One-pot synthesis of amino acid precursors with insoluble organic matter in planetesimals with aqueous activity. Sci. Adv. 3, e1602093 (2017)

L.P. Keller, S. Messenger, G.J. Flynn et al., The nature of molecular cloud material in interplanetary dust. Geochim. Cosmochim. Acta 68, 2577-2589 (2004)

J.F. Kerridge, Carbon, hydrogen and nitrogen in carbonaceous chondrites: abundances and isotopic compositions in bulk samples. Geochim. Cosmochim. Acta 49, 1707-1714 (1985)

J.F. Kerridge, S. Chang, R. Shipp, Isotopic characterisation of kerogen-like material in the Murchison carbonaceous chondrite. Geochim. Cosmochim. Acta 51, 2527-2540 (1987)

N. Khawaja, F. Postberg, J. Hillier et al., Low-mass nitrogen-, oxygen-bearing, and aromatic compounds in Enceladean ice grains. Mon. Not. R. Astron. Soc. 489, 5231-5243 (2019)

N. Kitadai, Predicting thermodynamic behaviors of non-protein amino acids as a function of temperature and pH. Orig. Life Evol. Biosph. 46, 3-18 (2016)

F. Kitajima, T. Nakamura, N. Takaoka et al., Evaluating the thermal metamorphism of CM chondrites by using the pyrolytic behavior of carbonaceous macromolecular matter. Geochim. Cosmochim. Acta 66, 163-172 (2002)

K. Kitazato, R.E. Milliken, T. Iwata et al., The surface composition of asteroid 162173 Ryugu from Hayabusa2 near-infrared spectroscopy. Science 364, 272-275 (2019)

H.P. Klein, The Viking biological experiments on Mars. Icarus 34, 666-674 (1978)

H.P. Klein, The Viking mission and the search for life on Mars. Rev. Geophys. Space Phys. 17, 1655-1662 (1979)

M. Komiya, A. Shimoyama, K. Harada, Examination of organic compounds from insoluble organic matter isolated from some Antarctic carbonaceous chondrites by heating experiments. Geochim. Cosmochim. Acta 57, 907-914 (1993)

R.V. Krishnamurthy, S. Epstein, J.R. Cronin et al., Isotopic and molecular analyses of hydrocarbons and monocarboxylic acids of the Murchison meteorite. Geochim. Cosmochim. Acta 56, 4045-4058 (1992) 
M. Kuga, B. Marty, Y. Marrocchi, L. Tissandier, Synthesis of refractory organic matter in the ionized gas phase of the solar nebula. Proc. Natl. Acad. Sci. 112, 7129-7134 (2015)

H.F. Levison, W.F. Bottke, M. Gounelle et al., Contamination of the asteroid belt by primordial transNeptunian objects. Nature 460, 364-366 (2009)

R.S. Lewis, T. Ming, J.F. Wacker et al., Interstellar diamonds in meteorites. Nature 326, 160-162 (1987)

J. Licandro, H. Campins, M. Kelley et al., (65) Cybele: detection of small silicate grains, water-ice, and organics. Astron. Astrophys. 525, A34 (2011)

K. Lodders, Solar system abundances and condensation temperatures of the elements. Astrophys. J. 591, 1220-1247 (2003)

Z. Martins, The nitrogen heterocycle content of meteorites and their significance for the origin of life. Life $\mathbf{8}$, 28-43 (2018)

Z. Martins, Organic molecules in meteorites and their astrobiological significance, in Handbook of Astrobiology, ed. by V.M. Kolb (CRC Press, Boca Raton, 2019), pp. 177-194. ISBN 9781138065123, CAT\# K33392.

Z. Martins, M.A. Sephton, Extraterrestrial amino acids, in Amino Acids, Peptides and Proteins in Organic Chemistry, ed. by A.B. Hughes (Wiley, New York, 2009), pp. 3-42

Z. Martins, J.S. Watson, M.A. Sephton et al., Free dicarboxylic and aromatic acids in the carbonaceous chondrites Murchison and Orgueil. Meteorit. Planet. Sci. 41, 1073-1080 (2006)

Z. Martins, B.A. Hofmann, E. Gnos et al., Amino acid composition, petrology, geochemistry, ${ }^{14} \mathrm{C}$ terrestrial age and oxygen isotopes of the Shisr 033 CR chondrite. Meteorit. Planet. Sci. 42, 1581-1595 (2007a)

Z. Martins, C.M.OD. Alexander, G.E. Orzechowska et al., Indigenous amino acids in primitive CR meteorites. Meteorit. Planet. Sci. 42, 2125-2136 (2007b)

Z. Martins, O. Botta, M.L. Fogel et al., Extraterrestrial nucleobases in the Murchison meteorite. Earth Planet. Sci. Lett. 270, 130-136 (2008)

Z. Martins, P. Modica, Z. Zanda et al., The amino acid and hydrocarbon contents of the Paris meteorite: insights into the most primitive CM chondrite. Meteorit. Planet. Sci. 50, 926-943 (2015)

G. Matrajt, S. Pizzarello, S. Taylor et al., Concentration and variability of the AIB amino acid in polar micrometeorites: implications for the exogenous delivery of amino acids to the primitive Earth. Meteorit. Planet. Sci. 39, 1849-1858 (2004)

G. Matrajt, G.M. Muñoz Caro, E. Dartois et al., FTIR analysis of the organics in IDPs: comparison with the IR spectra of the diffuse interstellar medium. Astron. Astrophys. 433, 979-995 (2005)

C. Meinert, U.J. Meierhenrich, A new dimension in separation science: comprehensive two-dimensional gas chromatography. Angew. Chem., Int. Ed. Engl. 51, 10460-10470 (2012)

C. Meinert, I. Myrgorodska, P. de Marcellus et al., Ribose and related sugars from ultraviolet irradiation of interstellar ice analogs. Science 352, 208-212 (2016)

S. Merouane, Z. Djouadi, L. D’Hendecourt, Relationship Between Organics and Silicates in Interplanetary Dust Particles, in 44th Lunar and Planetary Science Conference. LPI Contribution, No. 1719 (2003), p. 1981

S. Messenger, Identification of molecular-cloud material in interplanetary dust particles. Nature 404, 968-971 (2000)

S. Messenger, L.P. Keller, F.J. Stadermann et al., Samples of stars beyond the solar system: silicate grains in interplanetary dust. Science 300, 105-108 (2003)

T.J. Millar, A. Bennett, E. Herbst, Deuterium fractionation in dense interstellar clouds. Astrophys. J. 340, 906-920 (1989)

P. Modica, C. Meinert, P. de Marcellus et al., Enantiomeric excesses induced in amino acids by ultraviolet circularly polarized light irradiation of extraterrestrial ice analogs: a possible source of asymmetry for prebiotic chemistry. Astrophys. J. 788, 1-11 (2014)

P. Modica, Z. Martins, C. Meinert, B. Zanda, L.L.S. d'Hendecourt, The amino acid distribution in laboratory analogs of extraterrestrial organic matter: a comparison to CM chondrites. Astrophys. J. 865, 1-11 (2018)

M.J. Mumma, S.B. Charnley, The chemical composition of comets-emerging taxonomies and natal heritage. Annu. Rev. Astron. Astrophys. 49, 471-524 (2011)

G.M. Muñoz Caro, U.J. Meierhenrich, W.A. Schutte et al., Amino acids from ultraviolet irradiation of interstellar ice analogues. Nature 416, 403-406 (2002)

G.M. Muñoz Caro, G. Matrajt, E. Dartois et al., Nature and evolution of the dominant carbonaceous matter in interplanetary dust particles: effects of irradiation and identification with a type of amorphous carbon. Astron. Astrophys. 459, 147-159 (2006)

K. Nagao, R. Okazaki, T. Nakamura et al., Irradiation history of itokawa regolith material deduced from noble gases in the Hayabusa samples. Science 333, 1128-1131 (2011)

T. Nakamura, T. Noguchi, M. Tanaka et al., Itokawa dust particles: a direct link between S-type asteroids and ordinary chondrites. Science 333, 1113-1116 (2011) 
K. Nakamura-Messenger, S. Messenger, L.P. Keller et al., Organic globules in the Tagish Lake meteorite: remnants of the protosolar disk. Science 314, 1439-1442 (2006)

H. Naraoka, A. Shimoyama, K. Harada, Isotopic evidence from an Antarctica carbonaceous chondrite for two reaction pathways of extraterrestrial PAH formation. Earth Planet. Sci. Lett. 184, 1-7 (2000)

H. Naraoka, H. Mita, M. Komiya et al., A chemical sequence of macromolecular organic matter in the CM chondrites. Meteorit. Planet. Sci. 39, 401-406 (2004)

T. Noguchi, T. Nakamura, M. Kimura et al., Incipient space weathering observed on the surface of Itokawa dust particles. Science 333, 1121-1125 (2011)

M. Nuevo, S.N. Milam, S.A. Sandford et al., Formation of uracil from the ultraviolet photo-irradiation of pyrimidine in pure H2O ices. Astrobiology 9, 683-695 (2009)

M. Nuevo, J.H. Bredehöft, U.J. Meierhenrich et al., Urea, glycolic acid, and glycerol in an organic residue produced by ultraviolet irradiation of interstellar/pre-cometary ice analogs. Astrobiology 10, 245-256 (2010)

M. Nuevo, S.A. Sandford, G. Cooper, Sugars and sugar derivatives in residues produced from the UV irradiation of astrophysical ice analogs. LPI Contrib. 48, 2496 (2017)

F.-R. Orthous-Daunay, E. Quirico, P. Beck et al., Mid-infrared study of the molecular structure variability of insoluble organic matter from primitive chondrites. Icarus 223, 534-543 (2013)

E.E. Palmer, D.S. Lauretta, Aqueous alteration of kamacite in CM chondrites. Meteorit. Planet. Sci. 46, 1587-1607 (2011)

E.T. Peltzer, J.L. Bada, $\alpha$-Hydroxycarboxylic acids in the Murchison meteorite. Nature 272, 443-444 (1978)

C.M. Pieters, A. Nathues, G. Thangjam et al., Geologic constraints on the origin of red organic-rich material on Ceres. Meteorit. Planet. Sci. 53, 1983-1998 (2017)

F. Pietrucci, J.C. Aponte, R. Starr et al., Hydrothermal decomposition of amino acids and origins of prebiotic meteoritic organic compounds. ACS Earth Space Chem. 2, 588-598 (2018)

S. Pizzarello, T.L. Groy, Molecular asymmetry in extraterrestrial organic chemistry: an analytical perspective. Geochim. Cosmochim. Acta 75, 645-656 (2011)

S. Pizzarello, Y. Huang, Molecular and isotopic analyses of Tagish Lake alkyl dicarboxylic acids. Meteorit. Planet. Sci. 37, 687-696 (2002)

S. Pizzarello, Y. Huang, The deuterium enrichment of individual amino acids in carbonaceous meteorites: a case for the presolar distribution of biomolecule precursors. Geochim. Cosmochim. Acta 69, 599-605 (2005)

S. Pizzarello, C.T. Yarnes, Enantiomeric excesses of chiral amines in ammonia-rich carbonaceous meteorites. Earth Planet. Sci. Lett. 443, 176-184 (2016)

S. Pizzarello, R.V. Krishnamurthy, S. Epstein et al., Isotopic analyses of amino acids from the Murchison meteorite. Geochim. Cosmochim. Acta 55, 905-910 (1991)

S. Pizzarello, Y. Huang, L. Becker et al., The organic content of the Tagish Lake meteorite. Science 293, 2236-2239 (2001)

S. Pizzarello, M. Zolensky, K.A. Turk, Nonracemic isovaline in the Murchison meteorite: chiral distribution and mineral association. Geochim. Cosmochim. Acta 67, 1589-1595 (2003)

S. Pizzarello, Y. Huang, M. Fuller, The carbon isotopic distribution of Murchison amino acids. Geochim. Cosmochim. Acta 68, 4963-4969 (2004)

S. Pizzarello, G.W. Cooper, G.J. Flynn, The nature and distribution of the organic material in carbonaceous chondrites and interplanetary dust particles, in Meteorites and the Early Solar System II, ed. by D.S. Lauretta, H.Y. McSween Jr. (University of Arizona Press, Tucson, 2006), pp. 625-651

S. Pizzarello, Y. Wang, G.M. Chaban, A comparative study of the hydroxy acids from the Murchison, GRA 95229 and LAP 02342 meteorites. Geochim. Cosmochim. Acta 74, 6206-6217 (2010)

F. Postberg, S. Kempf, J.K. Hillier et al., The E-ring in the vicinity of Enceladus: II. Probing the moon's interior-the composition of E-ring particles. Icarus 193, 438-454 (2008)

F. Postberg, S. Kempf, J. Schmidt et al., Sodium salts in E-ring ice grains from an ocean below the surface of Enceladus. Nature 459, 1098 (2009)

E. Quirico, L. Bonal, Organic matter in interplanetary dusts and meteorites, in Biosignatures for Astrobiology. Advances in Astrobiology and Biogeophysics, ed. by B. Cavalazzi, F. Westall (Springer, Cham, 2019), pp. 23-50

E. Quirico, P.-I. Raynal, M. Bourot-Denise, Metamorphic grade of organic matter in six unequilibrated ordinary chondrites. Meteorit. Planet. Sci. 38, 795-811 (2003)

E. Quirico, L. Bonal, P. Beck et al., Prevalence and nature of heating processes in CM and C2-ungrouped chondrites as revealed by insoluble organic matter. Geochim. Cosmochim. Acta 241, 17-37 (2018)

M.A. Ratcliff, E.E. Medley, P.G. Simmonds, Pyrolysis of amino acids. Mechanistic considerations. J. Org. Chem. 39, 1481-1490 (1974)

L. Remusat, S. Derenne, F. Robert, New insight on aliphatic linkages in the macromolecular organic fraction of Orgueil and Murchison meteorites through ruthenium tetroxide oxidation. Geochim. Cosmochim. Acta 69, 4377-4386 (2005) 
A.S. Rivkin, J.P. Emery, Detection of ice and organics on an asteroidal surface. Nature 464, 1322-1323 (2010)

A.S. Rivkin et al., Hydrated minerals on asteroids: the astronomical record. Asteroids III 1, 235-253 (2002)

F. Robert, The D/H ratio in chondrites. Space Sci. Rev. 106, 87-101 (2003)

F. Robert, S. Epstein, The concentration and isotopic composition of hydrogen, carbon and nitrogen in carbonaceous meteorites. Geochim. Cosmochim. Acta 46, 81-95 (1982)

A. Rotundi, G.A. Baratta, J. Borg et al., Combined micro-Raman, micro-infrared, and field emission scanning electron microscope analyses of comet 81P/Wild 2 particles collected by Stardust. Meteorit. Planet. Sci. 43, 367-397 (2008)

S.A. Sandford, M.P. Bernstein, J.P. Dworkin, Assessment of the interstellar processes leading to deuterium enrichment in meteoritic organics. Meteorit. Planet. Sci. 36, 1117-1133 (2001)

S.A. Sandford et al., Organics captured from Comet $81 \mathrm{p} /$ Wild 2 by the stardust spacecraft. Science 314, 1720-1724 (2006)

P. Schmitt-Kopplin, Z. Gabelica, R.D. Gougeon et al., High molecular diversity of extraterrestrial organic matter in Murchison meteorite revealed 40 years after its fall. Proc. Natl. Acad. Sci. USA 107, 27632768 (2010)

M.A. Sephton, Organic compounds in carbonaceous meteorites. Natl. Prod. Rep. 19, 292-311 (2002)

M.A. Sephton, A.B. Verchovsky, P.A. Bland et al., Investigating the variations in carbon and nitrogen isotopes in carbonaceous chondrites. Geochim. Cosmochim. Acta 67, 2093-2108 (2003)

M.A. Sephton, G.D. Love, J.S. Watson et al., Hydropyrolysis of insoluble carbonaceous matter in the Murchison meteorite: new insights into its macromolecular structure. Geochim. Cosmochim. Acta 68, 13851393 (2004)

A. Shimoyama, H. Naraoka, N. Komiya et al., Analyses of carboxylic acids and hydrocarbons in Antarctic carbonaceous chondrites, Yamato-74662 and Yamato-793321. Geochem. J. 23, 181-193 (1989)

A. Shimoyama, S. Hagishita, K. Harada et al., Search for nucleic acid bases in carbonaceous chondrites from Antarctica. Geochem. J. 24, 343-348 (1990)

J.W. Smith, I.R. Kaplan, Endogenous carbon in carbonaceous meteorites. Science 167, 1367-1370 (1970)

N.A. Starkey, I.A. Franchi, C.M.OD. Alexander, A Raman spectroscopic study of organic matter in interplanetary dust particles and meteorites using multiple wavelength laser excitation. Meteorit. Planet. Sci. 48, 1800-1822 (2013)

N.A. Starkey, I.A. Franchi, M.R. Lee, Isotopic diversity in interplanetary dust particles and preservation of extreme ${ }^{16}$ O-depletion. Geochim. Cosmochim. Acta 142, 115-131 (2014)

S. Sugita et al., The geomorphology, color, and thermal properties of Ryugu: implications for parent-body processes. Science 364, 252 (2019)

D. Takir, J.P. Emery, H.Y. McSween et al., Nature and degree of aqueous alteration in CM and CI carbonaceous chondrites. Meteorit. Planet. Sci. 48, 1618-1637 (2013)

R. Terzieva, E. Herbst, The possibility of nitrogen isotopic fractionation in interstellar clouds. Mon. Not. R. Astron. Soc. 317, 563-568 (2000)

K.L. Thomas, G.E. Blanford, L.P. Keller et al., Carbon abundance and silicate mineralogy of anhydrous interplanetary dust particles. Geochim. Cosmochim. Acta 57, 1551-1566 (1993)

A.G.G.M. Tielens, Surface chemistry of deuterated molecules. Astron. Astrophys. 119, 177-184 (1983)

J.M. Trigo-Rodríguez, C.E. Moyano-Cambero, J. Llorca et al., UV to far-IR reflectance spectra of carbonaceous chondrites-I. implications for remote characterization of dark primitive asteroids targeted by sample-return missions. Mon. Not. R. Astron. Soc. 437, 227-240 (2014)

A. Tsuchiyama, M. Uesugi, T. Matsushima et al., Three-dimensional structure of Hayabusa samples: origin and evolution of Itokawa regolith. Science 333, 1125-1128 (2011)

M.J. Verdier-Paoletti et al., Oxygen isotope constraints on the alteration temperatures of CM chondrites. Earth Planet. Sci. Lett. 458, 273 (2016)

P. Vernazza, B. Zanda, T. Nakamura et al., The formation and evolution of ordinary chondrite parent bodies, in Asteroids IV, ed. by P. Michel, F.E. DeMeo, W.F. Bottke (University of Arizona Press, Tucson, 2015a), pp. 617-634

P. Vernazza, M. Marsset, P. Beck et al., Interplanetary dust particles as samples of icy asteroids. Astrophys. J. 806, 204-2013 (2015b)

V. Vinogradoff, C. Le Guillou, S. Bernard et al., Paris vs. Murchison: impact of hydrothermal alteration on organic matter in CM chondrites. Geochim. Cosmochim. Acta 212, 234-252 (2017)

S. Watanabe, M. Hirabayashi, N. Hirata et al., Hayabusa2 arrives at the carbonaceous asteroid 162173 Ryugu - a spinning top-shaped rubble pile. Science 364, 268-272 (2019)

M.K. Weisberg, T.J. McCoy, A.N. Krot, Systematics and evaluation of meteorite classification, in Meteorites and the Early Solar System II, ed. by D.S. Lauretta, H.Y. McSween (University of Arizona Press, Tucson, 2006), pp. 19-52 
K.C. Welten, K. Nishiizumi, M.W. Caffee, D.J. Hillegonds, J.A. Johnson, A.J.T. Jull, R. Wieler, L. Folco, Terrestrial ages, pairing, and concentration mechanism of Antarctic chondrites from Frontier Mountain, Northern Victoria Land. Meteorit. Planet. Sci. 41, 1081-1094 (2006)

B. Wopenka, Raman observations on individual interplanetary dust particles. Earth Planet. Sci. Lett. 88, 221231 (1988)

I.P. Wright, S. Sheridan, S.J. Barber et al., CHO-bearing organic compounds at the surface of 67P/Churyumov-Gerasimenko revealed by Ptolemy. Science 349, aab0673 (2015)

H. Yabuta, Solar system exploration: small bodies and their chemical and physical conditions, in Handbook of Astrobiology, ed. by V.M. Kolb (CRC Press, Boca Raton, 2019), pp. 23-42

H. Yabuta, H. Naraoka, K. Sakanishi et al., Solid-state ${ }^{13} \mathrm{C}$ NMR characterization of insoluble organic matter from Antarctic CM2 chondrites: evaluation of the meteoritic alteration level. Meteorit. Planet. Sci. 40, 779-787 (2005)

H. Yabuta, C.M.OD. Alexander, M.L. Fogel et al., A molecular and isotopic study of the macromolecular organic matter of the ungrouped C2 WIS 91600 and its relationship to Tagish Lake and PCA 91008. Meteorit. Planet. Sci. 45, 1446-1460 (2010)

H. Yabuta, T. Noguchi, S. Itoh et al., Formation of an ultracarbonaceous Antarctic micrometeorite through minimal aqueous alteration in a small porous icy body. Geochim. Cosmochim. Acta 214, 172-190 (2017)

J. Yang, S. Epstein, Interstellar organic matter in meteorites. Geochim. Cosmochim. Acta 47, 2199-2216 (1983)

J. Yang, S. Epstein, Relic interstellar grains in Murchison meteorite. Nature 311, 544-547 (1984)

G. Yuen, N. Blair, D.J. Des Marais et al., Carbon isotope composition of low molecular weight hydrocarbons and monocarboxylic acids from Murchison meteorite. Nature 307, 252-254 (1984)

H. Yurimoto, K. Abe, M. Abe et al., Oxygen isotopic compositions of asteroidal materials returned from Itokawa by the Hayabusa mission. Science 333, 1116-1118 (2011)

E. Zinner, S. Amari, B. Wopenka et al., Interstellar graphite in meteorites: isotopic compositions and structural properties of single graphite grains from Murchison. Meteoritics 30, 209 (1995)

M.E. Zolensky, W.L. Bourcier, J.L. Gooding, Aqueous alteration on the hydrous asteroids: results of Eq. 3/6 computer simulations. Icarus 78, 411-425 (1989) 